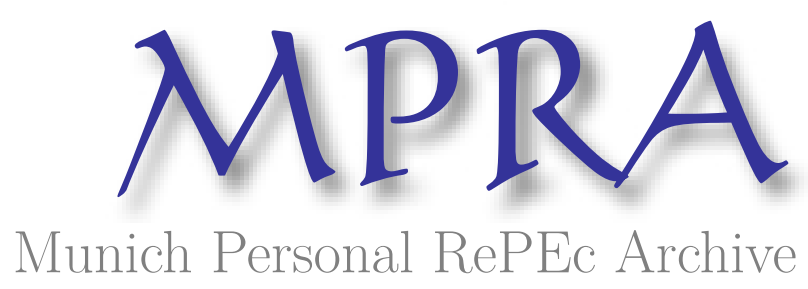

\title{
The Month-of-the-year Effect: Evidence from GARCH models in Fifty Five Stock Markets
}

Giovanis, Eleftherios

2009

Online at https://mpra.ub.uni-muenchen.de/22328/

MPRA Paper No. 22328, posted 25 Apr 2010 23:15 UTC 


\title{
The Month-of-the-year Effect: Evidence from GARCH models in Fifty Five Stock Markets
}

\author{
Eleftherios Giovanis
}

\begin{abstract}
This paper studies the month of the year effect, where January effect presents positive and the highest returns of the other months of the year. In order to investigate the specific calendar effect in global level, fifty five stock market indices from fifty one countries are examined. Symmetric GARCH models are applied and based on asymmetries tests asymmetric GARCH models are estimated. The main findings of this study is that a December effect is found on twenty stock markets, with higher returns on the specific month, while February effect is presented in nine stock markets, followed by January and April effects in seven and six stock markets respectively. These patterns provide positive and highest returns on the mentioned months, while a pattern where a specific month gives a persistence signal of negative returns couldn't be found.
\end{abstract}

Keywords: seasonality, stock returns, calendar effects, month of the year effect, asymmetric GARCH models, asymmetry tests, January effect

\section{Introduction}

January and the month of the year effect has been examined and investigated in various studies. Choudhry (2001) used monthly data for Index of Industrial shares in UK, Index of common stocks in Germany, Index of all industrial and public utilities and railroad common stocks to test the month-of-the year effect. Choudhry (2001) employed a MA(1)-GARCH-(1,1)-GJR model, including a moving average MA term to capture the effect of non-synchronous trading and found significant positive returns in January for UK and on January, April and August for USA, significant negative returns in March and July for UK, while significant positive returns in February, August, September and December and significant negative returns in June and October were found for Germany. Arsad and Coutts (1997) found that January displays significant positive returns after the introduction of capital gains tax in 1965 , while Aggarwal and Rivoli (1989) have found that January effects exists. 
Furthermore, other studies report positive and higher returns in both January and February (Mills et al., 2000; and Marquering, et al., 2006).

On the contrary other studies report different results. Szakmary and Kiefer (2004) found that the turn of the year effect in small capitalization stocks as the S\&P 400 Midcap and Russell 2000 indices, is eliminated by market participants. Generally January effect doesn't exist, but increased returns for small-cap stock indices on the last trading day of December are reported. Floros (2008) rejected January effect for three stock indices examined in Athens stock exchange market and higher returns over other months rather January are reported, but the estimated coefficients are statistically insignificant, except significant negative returns in June for all indices. Tonchev and Kim (2004) find that January effect exists only in the Czech Republic. Some evidence there is for the January effect for Slovenia and the half-month effect for the Czech Republic. Also they found a weak evidence for monthly seasonality in variance in all three countries. Giovanis (2009) examined fifty five stock markets and the January effect is rejected, as it is presented only in seven stock markets, while the most frequent significant higher monthly returns are reported in December of twelve stock markets. Since 1990 new approaches have been introduced in finance, which is the artificial intelligence, as neural networks and fuzzy logic. These approaches have been applied with success in finance, but they haven't been applied for the data mining of the calendar effects. Giovanis (2008) examined the month of the year effect in Athens stock exchange market and found higher returns in December.

The purpose of this paper is to investigate and test the January or the month of the year effect in a global level, without to be restricted in regional or national level, in order to examine if actually January presents the highest returns than the other months of the year, as also to recognize other monthly patterns which can be used for the 
optimum asset allocation with result the maximization of profits. Because each stock market behaves differently and presents different monthly patterns, the trading strategy should be formed in this way where the buy and sell signals and actions will be varied in each stock index.

\section{Methodology}

\subsection{The Regression Model}

The stock returns are defined by the following relation

$$
R_{t}=\log \left(P_{t}-P_{t-1}\right)
$$

Variable $P_{t}$ denotes the closed stock prices, while $P_{t-1}$ expresses the closed stock prices with one lag. For the examination of the month-of-the year effect the following regression is estimated:

$$
R_{t}=\sum_{i=1}^{12} \beta_{i} D_{i t}+\varepsilon_{t}
$$

,where $R_{t}$ is defined as in the relation (1), $D_{i t}$ represents the twelve dummy variables for twelve months, where $D_{l t}$ takes value 1 if returns belong in days of January and 0 otherwise, continuing at the last dummy variable $D_{12 t}$, which takes value 1 if stock returns belong in days of December and 0 otherwise and $\varepsilon_{t}$ is the disturbance term. The ordinary least squares method has been applied in all estimations, but the results are not reported, as in all cases heteroskedasticity, $\mathrm{ARCH}$ effects and autocorrelation were present. So for this reason is claimed that OLS estimations reports are not necessary, as the results are not reliable. 


\subsection{GARCH Methodology}

In order to estimate regression (2) GARCH models are applied. The first is the simple symmetric GARCH $(1,1)$ model which was proposed by Bollerslev (1986) and is defined as

$$
\varepsilon_{t} \sim\left(0, \sigma_{t}^{2}\right)
$$

, where $\varepsilon_{t}$ is the disturbance term of mean equation (2) and

$$
\sigma_{t}^{2}=\omega+a_{0} u_{t-1_{t}}^{2}+a_{1} \sigma_{t-1}^{2}
$$

The standard GARCH model is symmetric in its response to past innovations. Since good news and bad news may have different effects on the volatility two alternative GARCH models are considered in an attempt to capture the asymmetric nature of volatility responses. Since the symmetric GARCH model is unable to account for the leverage effects observed in stock returns, asymmetric GARCH models were proposed that enable conditional variance to respond asymmetrically to rises and falls in innovations

The other two GARCH models which are considered are the asymmetric EGARCH and GJR models. EGARCH $(1,1)$ model was proposed by Nelson (1991) and has the following form:

$$
\begin{aligned}
& \varepsilon_{t} \sim\left(0, \sigma_{t}{ }^{2}\right) \\
& \log \left(\sigma_{t}^{2}\right)=\omega+\log a_{0}\left({\sigma_{t-1}}^{2}\right)+a_{1} \frac{u_{t-1}}{\sqrt{\sigma_{t-1}^{2}}}+\gamma\left[\frac{\left|u_{t-1}\right|}{\sqrt{\sigma_{t-1}^{2}}}-\sqrt{\frac{2}{\pi}}\right]
\end{aligned}
$$

, where $\varepsilon_{t}$ is defined as in relation (3). We except for the asymmetries allowed finding a negative value for coefficient $\gamma$ if the relationship between volatility and returns is negative. More specifically it is expected $\gamma<0$, "good news" generate less volatility 
than "bad news", where $\gamma$ reflects the leverage effect. The second asymmetric GARCH model we estimate is GJR-GARCH $(1,1)$, which was proposed by Glosten et al. (1993)

$$
\sigma_{t}{ }^{2}=\omega+a_{0} u_{t-1}^{2}+a_{1} \sigma_{t-1}^{2}+\gamma u_{t-1}^{2} I_{t-1}
$$

$I_{t-1}$ is a dummy variable, where $I_{t-1}=1$ if $u_{t-1}^{2}<0$ and $I_{t-1}=0$ otherwise. Also for a leverage effect is expected that $\gamma>0$, so that the "bad news" have larger impacts, and is required that $\alpha_{1}+\gamma \geq 0$ and $\alpha_{1} \geq 0$ for non-negativity condition. It should be mentioned that the normal distribution, which was used by Engle (1982) is not followed, but in order the model to fully capture the excess kurtosis more fat tailed distributions that were proposed in the literature are used. Generally GARCH estimation behavior manifests itself as excessive kurtosis in residuals of the model. This property is known as fat tail or heavy-tailed distribution and means that extreme values have higher probabilities to occur. Since student- $t$ (Bollerslev, 1987) and the GED distribution (Nelson, 1991) have heavier tails than normal distribution, there is a better ability of generating large values (outliers) and therefore might be a better representative of conditional variance in the data. The $t$ distribution was chosen, as the estimated results between $t$ and GED distributions are almost the same.

It should be mentioned that we don't present the results of both asymmetric GARCH models in all stock markets, but each time we present only that we have chosen as the optimum. This choice is done based on Akaike and Schwartz information criteria, the Log-Likelihood statistic, as also based on which model is able to eliminate $\mathrm{ARCH}$ effects and autocorrelation. 


\subsection{Asymmetric tests}

Because the symmetric GARCH model is unable to capture for leverage effects, as it was mentioned previously we investigate if there are asymmetries in volatility of the calendar effects we examine. Here the methodology of the asymmetry tests proposed by Engle and NG (1993) is presented. We define $S_{t-1}$ as a dummy indicator taking value one if $u_{t-1}<0$ and zero otherwise. So the first test is the sign test and is defined by equation:

$$
{\hat{u_{t}}}^{2}=d_{0}+d_{1} S_{t-1}^{-}+e_{t}
$$

,where $e_{t}$ is an iid error term. If positive and negative shocks impact differently the upon the conditional variance then $d_{l}$ should be statistically significant. The second test is the negative sign bias and is defined as:

$$
\hat{u}_{t}^{2}=d_{0}+d_{1} S_{t-1}^{-} u_{t-1}+e_{t}
$$

, where $d_{1}$ should be also statistically significant. Then $S_{t-1}^{+}=1-S_{t-1}$ is defined, so that $S_{t-1}^{+}$picks out the observations with positive innovations and the positive sign bias test can be defined as:

$$
\hat{u}_{t}^{2}=d_{0}+d_{1} S_{t-1}^{+} u_{t-1}+e_{t}
$$

Engle and NG (1993) proposed a joint test for size and sign bias based on the following regression

$$
\hat{u}_{t}^{2}=d_{0}+d_{1} S_{t-1}^{-}+d_{2} S_{t-1}^{-} u_{t-1}+d_{3} S_{t-1}^{+} u_{t-1}+e_{t}
$$

, where significance of $d_{1}$ indicates the presence of sign bias, while on the other hand the significance $d_{2}$ or $d_{3}$ would suggest the presence of sign bias, where both the sign and the magnitude of the shock are important. The joint test is calculating by $N R^{2}$, 
where $N$ is the sample size, and asymptotically follows chi-square distribution with 3 degrees of freedom under the null hypothesis of no asymmetric effects. The null hypothesis is $H_{0}: d_{1}=d_{2}=d_{3}=0$.

\section{Data}

The analysis is conducted in terms of daily returns More specifically in table1 we present the countries, the indices symbols and the sources-websites where we found the data. The final period is 31 December 2008 for all series except from the starting period, where is shown in table 1.

(Table 1)

\section{Empirical results}

In table 2 the asymmetry tests of GARCH $(1,1)$ model are reported. We observe that the null hypothesis of the joint test (11) is rejected in all stock markets, except from stock markets in Luxemburg and Turkey. For this reason $\operatorname{GARCH}(1,1)$ is applied for these two stock markets. In table 3 the symmetric and asymmetric GARCH estimations of equation (2) are reported, where we note which asymmetric GARCH model is obtained in each stock market we examine. Table 4 reports the diagnostic tests of GARCH regressions.

The coefficients of GARCH equations are statically significant in the most cases. Furthermore, the coefficient $\gamma$ denoting the leverage effect is statistically significant and presents the expected and correct sign in all cases, except from the stock markets in Estonia, Latvia, Sri Lanka and Yugoslavia, where the coefficient $\gamma$ has the correct 
sign, but is insignificant, as well as in the case of Jordan, where the coefficient $\gamma$ presents the wrong sign as also is insignificant too.

From the overall results we observe that January effect is presented only in seven stock markets, which are in Malaysia, Pakistan, Peru, Singapore, Thailand and Dow Jones and Nasdaq-100 in USA. O the contrary we find a December effect, where the highest significant returns are reported in December, where the specific effects are presented in twenty stock markets. These are in Austria, Belgium, Brazil, Canada, Denmark, Estonia, Germany, India, Indonesia, Ireland, Luxemburg, Mexico, Netherlands, New Zealand, Philippine, Switzerland, Turkey, UK indices FTSE-100 and FTSE-250 and finally in Yugoslavia, where in Canada and New Zealand the highest returns are presented also in February and September respectively. Furthermore, a February effect is stronger than January, as it is presented in ten stock markets, Chile, Egypt, Finland, Hong Kong, Italy, Portugal, Russia, Spain, and Sweden including the stock market examined in Canada, as it was mentioned previously.

April effect is followed in Australia, China, Greece, Israel, Kuwait and S\&P 500 index in USA, while October presents the highest significant returns in the stock markets examined in Argentina, Croatia, and Norway. Some other weaker monthly anomalies are March, September and November effects presented in Japan, and France for March, Lithuania and Sri Lanka for September and South Korea and NY Composite for November. Finally, May exhibits higher significant returns in the stock market of Jordan, July in Latvia, June in Taiwan and August in Zambia.

On the contrary there aren't persistent anomalies and negative returns categorized in groups. For example it was expected that September might present negative returns in stock markets, but this is not happened as it is present only in China, while in the 
most cases returns in September are insignificant, while in few stock markets present positive significant returns, but not the lowest among the other months of the year.

\section{(Tables 2-4)}

\section{Conclusions}

The purpose of this paper was to examine the month of the year and the January effect. Because the most studies are restricted and repeated in major stock markets in the world, as Dow Jones Industrial and S\&P 500 in USA and FTSE-100 in UK among others, we tried to examine representative stock markets around the world and the analysis was not restricted in national and regional level or major stock markets, but was extended in global level. Generally, the results are mixed, but we conclude that January effects doesn't exist in global level and it is a very week calendar effect, as it is presented only in seven stock markets, while December presents higher returns in twelve stock markets. Furthermore, this study shows that the market efficiency hypothesis, always based on the month of the year effects, is violated, as in each stock market separately monthly patterns, with purpose the exploitation of profits, are formulated. 


\section{References}

Aggarwal, R., Rivoli, P. (1989). Seasonal and Day-of-the-Week Effects in Four Emerging Stock Markets, The Financial Review, Vol. 24 No. 7, pp. 541-550

Arsad, Z., Coutts, G.A. (1997). Security price anomalies in the London International Stock Exchange: a 60 year perspective, Applied Financial Economics, Vol. 7, pp. 455-464

Bollerslev, T. (1986). Generalized Autoregressive Conditional Heteroskedasticity. Journal of Econometrics, No. 3, pp. 307-327

Bollerslev T. (1987). A Conditionally Heteroskedastic Time Series Model for Speculative Prices and Rates of Return, The Review of Economics and Statistics, Vol. 69, pp. 542-547

Choudhry, T. (2001). Month of the year effect and January effect in Pre-WWI stock returns: Evidence from a non-linear GARCH model, International Journal of Finance and Economics, No. 6, pp. 1-11

Engle, R.F. (1982). Autoregressive Conditional Heteroscedasticity with Estimates of the Variance of United Kingdom Inflation. Econometrica, No. 50, pp. 987-1007

Engle, R. F., Ng., V. (1993). Measuring and Testing the Impact of News on Volatility. Journal of Finance, No. 48, pp. 1749-78

Floros, C., (2008). The monthly and trading month effects in Greek stock market returns: 1996-2002, Managerial Finance, Vol. 34 No. 7, pp. 453-464

Giovanis, E. (2008). Calendar Anomalies in Athens Exchange Stock Market - An Application of GARCH Models and the Neural Network Radial Basis Function, working paper

Giovanis, E. (2009). Calendar Effects in Fifty-five Stock Market Indices, Global Journal of Finance and Management, Vol. 1 No. 2, pp. 75-98

Glosten, L. R., Jagannathan, R. and Runkle, D.E. (1993). On the Relation between the Expected Value and the Volatility of the Nominal Excess Returns on Stocks. Journal of Finance, Vol. 48 No. 5, pp. 1779-1801

Marquering W., Nisser, J. and Valla, T. (2006). Disappearing anomalies: a dynamic analysis of the persistence of anomalies, Applied Financial Economics, No. 16, pp. 291-302

Mills, T. C., Siriopoulos, C., Markellos, R.N. and Harizanis, D. (2000). Seasonality in the Athens stock exchange, Applied Financial Economics, No. 10, pp. 137-142

Nelson, D. B. (1991). Conditional Heteroskedasticity In Asset Returns: A New Approach. Econometrica, No. 59, pp. 347-370

Szakmary, A..C. and Kiefer, D.B. (2004). The disappearing January/Turn of the year effect Evidence From Stock Index Futures and Cash Markets, The Financial Review, Vol. 24 No. 8, pp. 755-784

Tonchev D. and Kim, T.H. (2004). Calendar effects in Eastern European financial markets: evidence from the Czech Republic, Slovakia and Slovenia, Applied Financial Economics, No. 14, pp. 1035-1043 
Table 1 Stock Market Indices and estimating periods

\begin{tabular}{|c|c|c|c|}
\hline Countries & Period & Countries & Period \\
\hline Argentina (MERVAL INDEX) $^{1}$ & 9 October 1996 & Indonesia (JKSE Composite Index) ${ }^{1}$ & 2 July 1997 \\
\hline Australia (All ordinaries Index) ${ }^{2}$ & 9 January 2001 & Ireland (GENERAL INDEX) www.ise.ie & 4 January 1983 \\
\hline Austria (ATX INDEX) ${ }^{1}$ & 12 November 1992 & Israel (TA-100 INDEX) ${ }^{1}$ & 2 July 1997 \\
\hline Belgium (BFX INDEX) $^{1}$ & 14 February 2005 & Italy (MIBTEL INDEX) $^{1}$ & 4 January 2000 \\
\hline Brazil (IBOVESPA INDEX) $^{1}$ & 28 April 1993 & Japan(Nikkei 225) ${ }^{1}$ & 5 January 1984 \\
\hline $\begin{array}{l}\text { Canada (S\&P/TSX } \\
\text { Composite index) }\end{array}$ & 4 January 2000 & $\begin{array}{l}\text { Jordan (Weighted General Index) } \\
\underline{\text { www.ase.com.jo }}\end{array}$ & 4 January 1992 \\
\hline Chile (IPSA INDEX) $^{2}$ & 23 September 2003 & Kuwait (All Share Index) $)^{2}$ & 19 June 2001 \\
\hline China (Shanghai composite Index) $)^{2}$ & 4 July 1997 & $\begin{array}{l}\text { Latvia (OMX Riga) } \\
\text { www.baltic.omxnordicexchange.com }\end{array}$ & 4 January 2000 \\
\hline $\begin{array}{l}\text { Croatia (CROBEX INDEX) } \\
\underline{\text { www.zse.hr }}\end{array}$ & 3 January 1997 & $\begin{array}{l}\text { Lithuania (OMX Vilnius) } \\
\text { www.baltic.omxnordicexchange.com }\end{array}$ & 4 January 2000 \\
\hline 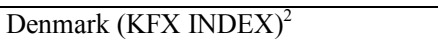 & 6 January 2000 & Luxemburg (LuxX INDEX) www.bourse.lu & 10 May 1988 \\
\hline Egypt (CCSI INDEX) ${ }^{1}$ & 3 July 1997 & Malaysia (KLSE INDEX) $^{1}$ & 6 December 1993 \\
\hline Estonia (OMX Tallinn) ${ }^{6}$ & 3 January 2000 & Mexico (IPC INDEX) $)^{1}$ & 11 November 1991 \\
\hline Finland (Helsinki General Index) $)^{2}$ & 4 July 1997 & Netherlands (AEX INDEX) ${ }^{1}$ & 13 October 1990 \\
\hline France $\left(\right.$ CAC 40 INDEX) ${ }^{1}$ & 2 March 1990 & $\begin{array}{l}\text { New Zealand (New Zealand } \\
\text { Stock Exchange } 50 \text { Index) })^{2}\end{array}$ & 5 May 2004 \\
\hline Germany (DAX INDEX) $^{1}$ & 27 November 1990 & Norway (OSEAX INDEX) $)^{1}$ & 8 February 2001 \\
\hline $\begin{array}{l}\text { Greece (GENERAL INDEX) } \\
\text { www.enet.gr }\end{array}$ & 5 Januaary 1998 & Pakistan (Karachi 100 Index) ${ }^{2}$ & 8 July 1997 \\
\hline Hong Kong (HANG SENG INDEX) $^{1}$ & 2 January 1987 & Peru (Lima General Index) $)^{2}$ & 4 May 1998 \\
\hline India (BSE SENSEX) ${ }^{1}$ & 2 January 1997 & Philippine (PSE Composite Index) ${ }^{2}$ & 7 July 1997 \\
\hline $\begin{array}{l}\text { Portugal (PSI GERAL INDEX) } \\
\text { www.euronext.com }\end{array}$ & 14 February 2005 & Turkey (ISTANBUL NAT-100) ${ }^{2}$ & 4 July 1997 \\
\hline $\begin{array}{l}\text { Russia Federation (RTSI INDEX) } \\
\text { www.rts.ru, }\end{array}$ & 4 September 1995 & UK (FTSE-100) $)^{2}$ & 3 April 1984 \\
\hline Singapore (STI INDEX)1 & 7 July 1997 & UK (FTSE-250) $)^{2}$ & 6 January 2000 \\
\hline $\begin{array}{l}\text { South Korea (KOSPI } \\
\text { Composite Index) } 1\end{array}$ & 2 July 1997 & USA (Dow Jones composite) $^{1}$ & 24 December 1980 \\
\hline Spain (IBEX 35)2 & 9 January 2002 & USA ( Nasdaq 100) ${ }^{1}$ & 8 February 1971 \\
\hline Sri Lanka (CSE All share Index) $)^{2}$ & 4 July 1997 & USA (NY composite) ${ }^{1}$ & 3 January 1966 \\
\hline $\begin{array}{l}\text { Sweden (SAX ALL SHARE } \\
\text { INDEX)2 }\end{array}$ & 9 January 2001 & USA (S\&P 500) ${ }^{1}$ & 4 January 1950 \\
\hline Swiss (SSMI INDEX) $^{1}$ & 12 November 1990 & $\begin{array}{l}\text { Yugoslavia (BELEX 15) } \\
\text { www.belex.co.yu }\end{array}$ & 5 October 2005 \\
\hline Taiwan (TSEC weighted index) ${ }^{1}$ & 3 July 1997 & $\begin{array}{l}\text { Zambia (LASI INDEX) } \\
\text { www.luse.co.zm }\end{array}$ & 2 January 2002 \\
\hline Thailand (SET INDEX) ${ }^{2}$ & 3 July 1997 & & \\
\hline
\end{tabular}

1.Source www.yahoofinance.com, 2. Source www.econstats.com 
Table 2. Asymmetric tests for the month-of-the-year effect

\begin{tabular}{|c|c|c|c|c|c|c|c|c|c|c|c|c|c|c|}
\hline Countries & Sign Bias & $\begin{array}{l}\text { Negative } \\
\text { Size Bias }\end{array}$ & $\begin{array}{c}\text { Positive } \\
\text { Size Bias }\end{array}$ & $\begin{array}{c}\text { Joint test } \\
\text { F-statistic }\end{array}$ & Countries & Sign Bias & $\begin{array}{l}\text { Negative } \\
\text { Size Bias }\end{array}$ & $\begin{array}{c}\text { Positive } \\
\text { Size Bias }\end{array}$ & $\begin{array}{c}\text { Joint test } \\
\text { F-statistic } \\
\end{array}$ & Countries & Sign Bias & $\begin{array}{l}\text { Negative } \\
\text { Size Bias }\end{array}$ & $\begin{array}{c}\text { Positive } \\
\text { Size Bias }\end{array}$ & $\begin{array}{c}\text { Joint test } \\
\text { F-statistic } \\
\end{array}$ \\
\hline ARGENTINA & $\begin{array}{l}7.60 \mathrm{e}-05 \\
(0.1225)\end{array}$ & $\begin{array}{c}-0.00885 \\
(0.000)\end{array}$ & $\begin{array}{l}0.00348 \\
(0.0224)\end{array}$ & $\begin{array}{l}12.167 \\
(0.000)\end{array}$ & IRELAND & $\begin{array}{c}-1.69 \mathrm{e}-05 \\
(0.9048)\end{array}$ & $\begin{array}{c}-0.0059 \\
(0.000)\end{array}$ & $\begin{array}{c}-0.003 \\
(0.0009)\end{array}$ & $\begin{array}{l}22.399 \\
(0.000)\end{array}$ & SINGAPORE & $\begin{array}{l}8.23 \mathrm{e}-07 \\
(0.9777)\end{array}$ & $\begin{array}{c}-0.00401 \\
(0.0024)\end{array}$ & $\begin{array}{c}0.0053 \\
(0.0001)\end{array}$ & $\begin{array}{c}7.896 \\
(0.000)\end{array}$ \\
\hline AUSTRALIA & $\begin{array}{l}4.80 \mathrm{e}-05 \\
(0.0737) \\
\end{array}$ & $\begin{array}{l}-0.0175 \\
(0.000) \\
\end{array}$ & $\begin{array}{r}-0.00192 \\
(0.2872) \\
\end{array}$ & $\begin{array}{l}22.545 \\
(0.000) \\
\end{array}$ & ISRAEL & $\begin{array}{l}3.19 \mathrm{e}-05 \\
(0.1279) \\
\end{array}$ & $\begin{array}{c}-0.00698 \\
(0.000) \\
\end{array}$ & $\begin{array}{c}-0.00270 \\
(0.0076) \\
\end{array}$ & $\begin{array}{l}18.659 \\
(0.000) \\
\end{array}$ & SOUTH KOREA & $\begin{array}{l}7.56 \mathrm{e}-05 \\
(0.0548) \\
\end{array}$ & $\begin{array}{r}-0.00374 \\
(0.0047) \\
\end{array}$ & $\begin{array}{l}0.00091 \\
(0.4812) \\
\end{array}$ & $\begin{array}{c}4.076 \\
(0.0067) \\
\end{array}$ \\
\hline AUSTRIA & $\begin{array}{l}4.02 \mathrm{e}-05 \\
(0.0007)\end{array}$ & $\begin{array}{c}-0.00462 \\
(0.000)\end{array}$ & $\begin{array}{c}-0.0029 \\
(0.000)\end{array}$ & $\begin{array}{l}20.557 \\
(0.000)\end{array}$ & ITALY & $\begin{array}{l}3.73 \mathrm{e}-05 \\
(0.0060)\end{array}$ & $\begin{array}{r}-0.00317 \\
(0.0002)\end{array}$ & $\begin{array}{c}-0.00369 \\
(0.0131)\end{array}$ & $\begin{array}{l}13.752 \\
(0.000)\end{array}$ & SPAIN & $\begin{array}{l}4.78 \mathrm{e}-05 \\
(0.0024)\end{array}$ & $\begin{array}{c}-0.00383 \\
(0.000)\end{array}$ & $\begin{array}{c}-0.00289 \\
(0.0006)\end{array}$ & $\begin{array}{c}14.616 \\
(0.0000)\end{array}$ \\
\hline BELGIUM & $\begin{array}{l}2.85 \mathrm{e}-05 \\
(0.3246)\end{array}$ & $\begin{array}{l}-0.00516 \\
(0.0024)\end{array}$ & $\begin{array}{l}-0.00461 \\
(0.0140)\end{array}$ & $\begin{array}{c}5.627 \\
(0.0008)\end{array}$ & JAPAN & $\begin{array}{l}2.77 \mathrm{e}-05 \\
(0.0580)\end{array}$ & $\begin{array}{c}-0.00324 \\
(0.000)\end{array}$ & $\begin{array}{c}-0.00442 \\
(0.000)\end{array}$ & $\begin{array}{l}19.422 \\
(0.000)\end{array}$ & SRI LANKA & $\begin{array}{l}1.91 \mathrm{e}-05 \\
(0.4876)\end{array}$ & $\begin{array}{l}-0.00531 \\
(0.0006)\end{array}$ & $\begin{array}{l}0.00257 \\
(0.1371)\end{array}$ & $\begin{array}{c}4.809 \\
(0.0024)\end{array}$ \\
\hline BRAZIL & $\begin{array}{c}-6.51 \mathrm{e}-05 \\
(0.3591)\end{array}$ & $\begin{array}{l}-0.0114 \\
(0.000)\end{array}$ & $\begin{array}{l}-0.0085 \\
(0.000)\end{array}$ & $\begin{array}{l}18.726 \\
(0.000)\end{array}$ & JORDAN & $\begin{array}{r}-1.81 \mathrm{e}-05 \\
(0.0178)\end{array}$ & $\begin{array}{c}-0.00464 \\
(0.000)\end{array}$ & $\begin{array}{l}-0.00018 \\
(0.7334)\end{array}$ & $\begin{array}{l}21.625 \\
(0.000)\end{array}$ & SWEDEN & $\begin{array}{l}3.19 \mathrm{e}-05 \\
(0.0848)\end{array}$ & $\begin{array}{l}-0.00291 \\
(0.0023)\end{array}$ & $\begin{array}{l}-0.00374 \\
(0.0001)\end{array}$ & $\begin{array}{c}9.046 \\
(0.000)\end{array}$ \\
\hline CANADA & $\begin{array}{l}3.32 \mathrm{e}-05 \\
(0.0236)\end{array}$ & $\begin{array}{l}-0.00273 \\
(0.0038)\end{array}$ & $\begin{array}{l}-0.00198 \\
(0.0448)\end{array}$ & $\begin{array}{c}5.708 \\
(0.0007)\end{array}$ & KUWAIT & $\begin{array}{l}2.45 \mathrm{e}-05 \\
(0.0234)\end{array}$ & $\begin{array}{l}-0.0108 \\
(0.000)\end{array}$ & $\begin{array}{l}0.00012 \\
(0.8738)\end{array}$ & $\begin{array}{l}66.566 \\
(0.000)\end{array}$ & SWITZERLAND & $\begin{array}{l}2.40 \mathrm{e}-05 \\
(0.0157)\end{array}$ & $\begin{array}{c}-0.00479 \\
(0.000)\end{array}$ & $\begin{array}{l}-0.00185 \\
(0.0022)\end{array}$ & $\begin{array}{l}25.502 \\
(0.000)\end{array}$ \\
\hline CHILE & $\begin{array}{l}2.07 \mathrm{e}-05 \\
(0.0974) \\
\end{array}$ & $\begin{array}{c}-0.00663 \\
(0.000) \\
\end{array}$ & $\begin{array}{r}-0.00332 \\
(0.0008) \\
\end{array}$ & $\begin{array}{l}23.080 \\
(0.000) \\
\end{array}$ & LATVIA & $\begin{array}{l}2.42 \mathrm{e}-05 \\
(0.5921) \\
\end{array}$ & $\begin{array}{r}-0.0197 \\
(0.000) \\
\end{array}$ & $\begin{array}{l}0.00686 \\
(0.0014) \\
\end{array}$ & $\begin{array}{l}35.090 \\
(0.000)\end{array}$ & TAIWAN & $\begin{array}{l}1.81 \mathrm{e}-05 \\
(0.3937) \\
\end{array}$ & $\begin{array}{c}-0.00448 \\
(0.000) \\
\end{array}$ & $\begin{array}{r}-0.00083 \\
(0.3579) \\
\end{array}$ & $\begin{array}{c}8.532 \\
(0.000) \\
\end{array}$ \\
\hline CHINA & $\begin{array}{l}2.97 \mathrm{e}-05 \\
(0.2520)\end{array}$ & $\begin{array}{r}-0.00505 \\
(0.0001)\end{array}$ & $\begin{array}{l}-0.00232 \\
(0.0312)\end{array}$ & $\begin{array}{c}6.717 \\
(0.0002)\end{array}$ & LITHUANIA & $\begin{array}{l}1.69 \mathrm{e}-05 \\
(0.1898)\end{array}$ & $\begin{array}{c}-0.00499 \\
(0.000)\end{array}$ & $\begin{array}{l}0.00033 \\
(0.7284)\end{array}$ & $\begin{array}{c}9.086 \\
(0.000)\end{array}$ & THAILAND & $\begin{array}{l}-0.00011 \\
(0.0164)\end{array}$ & $\begin{array}{l}-0.00103 \\
(0.4977)\end{array}$ & $\begin{array}{l}0.0111 \\
(0.000)\end{array}$ & $\begin{array}{l}18.765 \\
(0.000)\end{array}$ \\
\hline CROATIA & $\begin{array}{r}-1.64 \mathrm{e}-05 \\
(0.7478)\end{array}$ & $\begin{array}{l}0.00265 \\
(0.1889)\end{array}$ & $\begin{array}{l}-0.0171 \\
(0.0157)\end{array}$ & $\begin{array}{l}19.460 \\
(0.000)\end{array}$ & LUXEMBURG & $\begin{array}{l}0.00015 \\
(0.3053)\end{array}$ & $\begin{array}{c}-0.00714 \\
(0.000)\end{array}$ & $\begin{array}{l}-0.00248 \\
(0.0131)\end{array}$ & $\begin{array}{c}1.747 \\
(0.1550)\end{array}$ & TURKEY & $\begin{array}{c}-1.47 \mathrm{e}-05 \\
(0.8572)\end{array}$ & $\begin{array}{l}-0.00208 \\
(0.3094)\end{array}$ & $\begin{array}{c}-3.30 \mathrm{e}-05 \\
(0.9873)\end{array}$ & $\begin{array}{c}0.3563 \\
(0.7846)\end{array}$ \\
\hline DENMARK & $\begin{array}{l}3.75 \mathrm{e}-05 \\
(0.0009)\end{array}$ & $\begin{array}{c}-0.00126 \\
(0.0715)\end{array}$ & $\begin{array}{r}-0.00174 \\
(0.0202)\end{array}$ & $\begin{array}{c}7.042 \\
(0.0001)\end{array}$ & MALAYSIA & $\begin{array}{l}-1.8 \mathrm{e}-05 \\
(0.7263)\end{array}$ & $\begin{array}{c}0.0218 \\
(0.000)\end{array}$ & $\begin{array}{l}0.0102 \\
(0.000)\end{array}$ & $\begin{array}{l}33.061 \\
(0.000)\end{array}$ & UK-FTSE 100 & $\begin{array}{l}2.08 \mathrm{e}-05 \\
(0.0180)\end{array}$ & $\begin{array}{c}-0.00475 \\
(0.000)\end{array}$ & $\begin{array}{c}-0.00347 \\
(0.000)\end{array}$ & $\begin{array}{l}35.628 \\
(0.000)\end{array}$ \\
\hline EGYPT & $\begin{array}{l}1.70 \mathrm{e}-05 \\
(0.2942)\end{array}$ & $\begin{array}{c}-0.00793 \\
(0.000)\end{array}$ & $\begin{array}{r}-0.00277 \\
(0.0154)\end{array}$ & $\begin{array}{l}20.584 \\
(0.000) \\
\end{array}$ & MEXICO & $\begin{array}{l}9.05 \mathrm{e}-06 \\
(0.6789)\end{array}$ & $\begin{array}{l}-0.0067 \\
(0.000)\end{array}$ & $\begin{array}{c}-0.0025 \\
(0.0075)\end{array}$ & $\begin{array}{l}18.823 \\
(0.000)\end{array}$ & UK-FTSE 250 & $\begin{array}{l}3.87 \mathrm{e}-05 \\
(0.0006)\end{array}$ & $\begin{array}{c}-0.00324 \\
(0.0001)\end{array}$ & $\begin{array}{r}-0.00242 \\
(0.0032)\end{array}$ & $\begin{array}{l}12.395 \\
(0.000)\end{array}$ \\
\hline ESTONIA & $\begin{array}{l}1.00 \mathrm{e}-05 \\
(0.4411)\end{array}$ & $\begin{array}{l}-0.0056 \\
(0.000)\end{array}$ & $\begin{array}{l}0.00155 \\
(0.1474)\end{array}$ & $\begin{array}{l}11.224 \\
(0.000)\end{array}$ & NETHERLANDS & $\begin{array}{l}3.87 \mathrm{e}-05 \\
(0.0116)\end{array}$ & $\begin{array}{l}-0.0039 \\
(0.000)\end{array}$ & $\begin{array}{l}-0.0033 \\
(0.000)\end{array}$ & $\begin{array}{l}16.089 \\
(0.000)\end{array}$ & $\begin{array}{l}\text { US DOW JONES } \\
\text { COMPOSITE }\end{array}$ & $\begin{array}{l}2.43 \mathrm{e}-05 \\
(0.1231)\end{array}$ & $\begin{array}{c}-0.00825 \\
(0.000)\end{array}$ & $\begin{array}{l}-0.0003 \\
(0.7843)\end{array}$ & $\begin{array}{l}17.730 \\
(0.000)\end{array}$ \\
\hline FINLAND & $\begin{array}{l}8.89 \mathrm{e}-05 \\
(0.0672)\end{array}$ & $\begin{array}{l}-0.0061 \\
(0.0003)\end{array}$ & $\begin{array}{c}-0.00392 \\
(0.0156)\end{array}$ & $\begin{array}{c}7.697 \\
(0.000) \\
\end{array}$ & NEW ZEALAND & $\begin{array}{l}1.19 \mathrm{e}-05 \\
(0.0295)\end{array}$ & $\begin{array}{r}-0.00129 \\
(0.0232)\end{array}$ & $\begin{array}{l}0.00038 \\
(0.5178)\end{array}$ & $\begin{array}{c}3.474 \\
(0.0156)\end{array}$ & US NASDAQ 100 & $\begin{array}{c}4.66 \mathrm{e}-05 \\
(0.000)\end{array}$ & $\begin{array}{c}-0.0056 \\
(0.000)\end{array}$ & $\begin{array}{l}-0.0055 \\
(0.000)\end{array}$ & $\begin{array}{l}64.245 \\
(0.000) \\
\end{array}$ \\
\hline FRANCE & $\begin{array}{l}1.68 \mathrm{e}-05 \\
(0.1650)\end{array}$ & $\begin{array}{r}-0.00249 \\
(0.0001)\end{array}$ & $\begin{array}{r}-0.00139 \\
(0.0273)\end{array}$ & $\begin{array}{c}7.925 \\
(0.000)\end{array}$ & NORWAY & $\begin{array}{l}6.37 \mathrm{e}-05 \\
(0.0009)\end{array}$ & $\begin{array}{c}-0.00833 \\
(0.000)\end{array}$ & $\begin{array}{c}-0.00519 \\
(0.000)\end{array}$ & $\begin{array}{l}35.364 \\
(0.000)\end{array}$ & $\begin{array}{c}\text { US NEW YORK } \\
\text { COMPOSITE }\end{array}$ & $\begin{array}{l}1.23 \mathrm{e}-05 \\
(0.1643)\end{array}$ & $\begin{array}{c}-0.00498 \\
(0.000)\end{array}$ & $\begin{array}{c}-0.00264 \\
(0.0001)\end{array}$ & $\begin{array}{l}22.489 \\
(0.000)\end{array}$ \\
\hline GERMANY & $\begin{array}{l}3.70 \mathrm{e}-05 \\
(0.0071)\end{array}$ & $\begin{array}{c}-0.00415 \\
(0.000)\end{array}$ & $\begin{array}{l}-0.00248 \\
(0.0004)\end{array}$ & $\begin{array}{l}18.621 \\
(0.000)\end{array}$ & PAKISTAN & $\begin{array}{c}0.000118 \\
(0.000)\end{array}$ & $\begin{array}{c}-0.00627 \\
(0.000)\end{array}$ & $\begin{array}{l}-0.00224 \\
(0.0419)\end{array}$ & $\begin{array}{l}19.396 \\
(0.000)\end{array}$ & US -S\&P 500 & $\begin{array}{l}1.63 \mathrm{e}-05 \\
(0.0414)\end{array}$ & $\begin{array}{c}-0.00677 \\
(0.000)\end{array}$ & $\begin{array}{l}-0.00151 \\
(0.0110)\end{array}$ & $\begin{array}{l}39.958 \\
(0.000)\end{array}$ \\
\hline GREECE & $\begin{array}{l}-0.0001 \\
(0.2167)\end{array}$ & $\begin{array}{l}-0.0106 \\
(0.0002)\end{array}$ & $\begin{array}{l}-0.0548 \\
(0.000)\end{array}$ & $\begin{array}{l}110.122 \\
(0.000)\end{array}$ & PERU & $\begin{array}{l}2.04 \mathrm{e}-05 \\
(0.2704)\end{array}$ & $\begin{array}{l}-0.0101 \\
(0.000)\end{array}$ & $\begin{array}{l}-0.00293 \\
(0.0043)\end{array}$ & $\begin{array}{l}34.555 \\
(0.000)\end{array}$ & YUGOSLAVIA & $\begin{array}{c}-1.98 \mathrm{e}-05 \\
(0.7487)\end{array}$ & $\begin{array}{l}-0.00995 \\
(0.0005)\end{array}$ & $\begin{array}{l}0.0146 \\
(0.000)\end{array}$ & $\begin{array}{l}11.314 \\
(0.000)\end{array}$ \\
\hline $\begin{array}{l}\text { HONG } \\
\text { KONG }\end{array}$ & $\begin{array}{l}0.000105 \\
(0.1318)\end{array}$ & $\begin{array}{l}-0.0390 \\
(0.000)\end{array}$ & $\begin{array}{l}-0.0075 \\
(0.0064)\end{array}$ & $\begin{array}{l}58.649 \\
(0.000)\end{array}$ & PHILLIPINE & $\begin{array}{l}1.56 \mathrm{e}-06 \\
(0.9579)\end{array}$ & $\begin{array}{l}-0.00387 \\
(0.0035)\end{array}$ & $\begin{array}{l}0.00529 \\
(0.0001)\end{array}$ & $\begin{array}{c}7.657 \\
(0.000)\end{array}$ & ZAMBIA & $\begin{array}{c}-0.00021 \\
(0.000)\end{array}$ & $\begin{array}{c}0.00544 \\
(0.000)\end{array}$ & $\begin{array}{l}-0.0103 \\
(0.000)\end{array}$ & $\begin{array}{l}34.558 \\
(0.000)\end{array}$ \\
\hline INDIA & $\begin{array}{l}7.52 \mathrm{e}-05 \\
(0.0017)\end{array}$ & $\begin{array}{c}-0.00795 \\
(0.000)\end{array}$ & $\begin{array}{r}-0.00393 \\
(0.0001)\end{array}$ & $\begin{array}{l}28.850 \\
(0.000)\end{array}$ & PORTUGAL & $\begin{array}{l}3.19 \mathrm{e}-05 \\
(0.0922)\end{array}$ & $\begin{array}{c}-0.00465 \\
(0.000)\end{array}$ & $\begin{array}{c}-0.00608 \\
(0.2413)\end{array}$ & $\begin{array}{l}(0.000) \\
(0.000)\end{array}$ & & & & & \\
\hline INDONESIA & $\begin{array}{l}7.38 \mathrm{e}-05 \\
(0.1347)\end{array}$ & $\begin{array}{l}-0.0085 \\
(0.000)\end{array}$ & $\begin{array}{l}0.00368 \\
(0.0157)\end{array}$ & $\begin{array}{l}11.497 \\
(0.000)\end{array}$ & RUSSIA & $\begin{array}{c}0.000171 \\
(0.0285)\end{array}$ & $\begin{array}{c}-0.0187 \\
(0.0015)\end{array}$ & $\begin{array}{l}0.00226 \\
(0.000)\end{array}$ & $\begin{array}{l}29.452 \\
(0.000)\end{array}$ & & & & & \\
\hline
\end{tabular}

P-values in parentheses 
Table 3. GARCH estimations of equation (2)

\begin{tabular}{|c|c|c|c|c|c|c|c|c|c|c|c|c|c|c|c|c|}
\hline Countries & $\boldsymbol{\beta}_{1}$ & $\boldsymbol{\beta}_{2}$ & $\beta_{3}$ & $\beta_{4}$ & $\beta_{5}$ & $\beta_{6}$ & $\beta_{7}$ & $\beta_{8}$ & $\beta_{9}$ & $\beta_{10}$ & $\beta_{11}$ & $\beta_{12}$ & (1) & $\alpha_{0}$ & $\gamma$ & $\alpha_{1}$ \\
\hline $\begin{array}{l}\text { ARGENTINA } \\
\text { GJR-GARCH }\end{array}$ & $\begin{array}{c}0.00161 \\
(0.00098) \\
{[1.640]}\end{array}$ & $\begin{array}{c}0.00197 \\
(0.00098) \\
{[2.003]^{* *}}\end{array}$ & $\begin{array}{c}-0.00034 \\
(0.00095) \\
{[-0.360]}\end{array}$ & $\begin{array}{c}0.00058 \\
(0.00098) \\
{[0.588]}\end{array}$ & $\begin{array}{c}0.00063 \\
(0.00110) \\
{[0.578]}\end{array}$ & $\begin{array}{c}-0.00043 \\
(0.00097) \\
{[-0.445]}\end{array}$ & $\begin{array}{c}0.00051 \\
(0.00101) \\
{[0.504]}\end{array}$ & $\begin{array}{c}-0.00098 \\
(0.00097) \\
{[-1.007]}\end{array}$ & $\begin{array}{c}0.00174 \\
(0.00093) \\
{[1.885]^{* * *}}\end{array}$ & $\begin{array}{c}0.00206 \\
(0.00090) \\
{[2.284]^{* *}}\end{array}$ & $\begin{array}{c}0.00131 \\
(0.00096) \\
{[1.361]}\end{array}$ & $\begin{array}{c}0.00189 \\
(0.00109) \\
{[1.730]^{* * *}}\end{array}$ & $\begin{array}{c}1.50 \mathrm{e}-05 \\
(2.99 \mathrm{e}-06) \\
{[5.033]^{*}}\end{array}$ & $\begin{array}{c}0.0588 \\
(0.0154) \\
{[3.825]^{*}}\end{array}$ & $\begin{array}{c}0.1151 \\
(0.0232) \\
{[4.974]^{*}}\end{array}$ & $\begin{array}{c}0.8531 \\
(0.0170) \\
{[50.259]^{*}}\end{array}$ \\
\hline $\begin{array}{c}\text { AUSTRALIA } \\
\text { EGARCH }\end{array}$ & $\begin{array}{c}0.00068 \\
(0.00028) \\
{[2.355]^{* *}}\end{array}$ & $\begin{array}{c}0.00040 \\
(0.00030) \\
{[1.299]}\end{array}$ & $\begin{array}{c}0.00060 \\
(0.00029) \\
{[2.010]^{* *}}\end{array}$ & $\begin{array}{c}0.00113 \\
(0.00031) \\
{[3.640]^{*}}\end{array}$ & $\begin{array}{c}0.00025 \\
(0.00030) \\
{[0.821]}\end{array}$ & $\begin{array}{c}-3.46 \mathrm{e}-05 \\
(0.00030) \\
{[-0.112]}\end{array}$ & $\begin{array}{c}0.00081 \\
(0.00028) \\
{[2.822]^{*}}\end{array}$ & $\begin{array}{c}0.00063 \\
(0.00030) \\
{[2.127]^{* *}}\end{array}$ & $\begin{array}{c}0.00035 \\
(0.00029) \\
{[1.234]}\end{array}$ & $\begin{array}{c}0.00105 \\
(0.00030) \\
{[3.492]^{*}}\end{array}$ & $\begin{array}{c}1.26 \mathrm{e}-06 \\
(0.00033) \\
{[0.003]}\end{array}$ & $\begin{array}{c}0.00097 \\
(0.00033) \\
{[2.895]^{*}}\end{array}$ & $\begin{array}{c}-0.4608 \\
(0.0558) \\
{[-8.251]^{*}}\end{array}$ & & $\begin{array}{c}-0.0585 \\
(0.0083) \\
{[-7.020]^{*}}\end{array}$ & $\begin{array}{c}0.9656 \\
(0.0051) \\
{[187.25]^{*}}\end{array}$ \\
\hline $\begin{array}{c}\text { AUSTRIA } \\
\text { GJR-GARCH }\end{array}$ & $\begin{array}{c}0.00134 \\
(0.00047) \\
{[2.877]^{*}}\end{array}$ & $\begin{array}{c}0.00156 \\
(0.00047) \\
{[3.329]^{*}}\end{array}$ & $\begin{array}{c}-0.00016 \\
(0.00049) \\
{[-0.330]}\end{array}$ & $\begin{array}{c}0.00120 \\
(0.00048) \\
{[2.504]^{* *}}\end{array}$ & $\begin{array}{c}0.00082 \\
(0.00047) \\
{[1.744]^{* * *}}\end{array}$ & $\begin{array}{c}0.00020 \\
(0.00049) \\
{[0.412]}\end{array}$ & $\begin{array}{c}0.00017 \\
(0.00045) \\
{[0.382]}\end{array}$ & $\begin{array}{c}0.00016 \\
(0.00051) \\
{[0.319]} \\
\end{array}$ & $\begin{array}{c}-0.00069 \\
(0.00048) \\
{[-1.421]}\end{array}$ & $\begin{array}{c}0.00093 \\
(0.00052) \\
{[1.790]^{* * *}}\end{array}$ & $\begin{array}{c}0.00084 \\
(0.00049) \\
{[1.705]^{* * *}}\end{array}$ & $\begin{array}{c}0.00175 \\
(0.00050) \\
{[3.520]^{*}} \\
\end{array}$ & $\begin{array}{c}4.20 \mathrm{e}-06 \\
(7.72 \mathrm{e}-07) \\
{[5.447]^{*}}\end{array}$ & & $\begin{array}{c}0.0822 \\
(0.0171) \\
{[4.817]^{*}}\end{array}$ & $\begin{array}{c}0.8710 \\
(0.0146) \\
{[59.492]^{*}}\end{array}$ \\
\hline $\begin{array}{l}\text { BELGIUM } \\
\text { EGARCH }\end{array}$ & $\begin{array}{c}0.00129 \\
(0.00060) \\
{[2.138]^{* *}}\end{array}$ & $\begin{array}{c}0.00124 \\
(0.00062) \\
{[2.164]^{* *}}\end{array}$ & $\begin{array}{c}-0.00078 \\
(0.00056) \\
{[1.387]}\end{array}$ & $\begin{array}{c}0.00023 \\
(0.00059) \\
{[0.388]}\end{array}$ & $\begin{array}{c}-0.00032 \\
(0.00065) \\
{[-0.499]}\end{array}$ & $\begin{array}{c}-0.00066 \\
(0.00071) \\
{[-0.928]}\end{array}$ & $\begin{array}{c}0.00056 \\
(0.00064) \\
{[0.874]}\end{array}$ & $\begin{array}{c}-0.00046 \\
(0.00063) \\
{[-0.734]}\end{array}$ & $\begin{array}{c}0.00092 \\
(0.00061) \\
{[1.520]}\end{array}$ & $\begin{array}{c}0.00013 \\
(0.00061) \\
{[0.212]}\end{array}$ & $\begin{array}{c}-0.00023 \\
(0.00060) \\
{[0.380]}\end{array}$ & $\begin{array}{c}0.00165 \\
(0.00057) \\
{[2.894]^{*}}\end{array}$ & & $\begin{array}{c}0.0457 \\
(0.0262) \\
{[1.714]^{* * *}}\end{array}$ & & $\begin{array}{c}0.9815 \\
(0.0044) \\
{[221.33]^{*}}\end{array}$ \\
\hline $\begin{array}{c}\text { BRAZIL } \\
\text { GJR-GARCH }\end{array}$ & $\begin{array}{c}0.00201 \\
(0.00103) \\
{[1.945]^{* * *}}\end{array}$ & $\begin{array}{c}0.00153 \\
(0.00119) \\
{[1.279]}\end{array}$ & $\begin{array}{c}0.00064 \\
(0.00106) \\
{[0.601]}\end{array}$ & $\begin{array}{c}0.00161 \\
(0.00106) \\
{[1.521]} \\
\end{array}$ & $\begin{array}{c}0.00233 \\
(0.00096) \\
{[2.427]^{* *}}\end{array}$ & $\begin{array}{c}0.00090 \\
(0.00102) \\
{[0.886]}\end{array}$ & $\begin{array}{c}0.00061 \\
(0.00095) \\
{[0.641]} \\
\end{array}$ & $\begin{array}{c}0.00081 \\
(0.00099) \\
{[0.818]} \\
\end{array}$ & $\begin{array}{c}0.00132 \\
(0.00104) \\
{[1.265]}\end{array}$ & $\begin{array}{c}0.00129 \\
(0.00098) \\
{[1.318]}\end{array}$ & $\begin{array}{c}0.00274 \\
(0.00112) \\
{[2.443]^{* *}} \\
\end{array}$ & $\begin{array}{c}0.00327 \\
(0.00104) \\
{[3.138]^{*}}\end{array}$ & $\begin{array}{c}9.79 \mathrm{e}-06 \\
(2.19 \mathrm{e}-06) \\
{[4.465]^{*}}\end{array}$ & & & $\begin{array}{c}0.8789 \\
(0.0126) \\
{[69.990]^{*}}\end{array}$ \\
\hline $\begin{array}{l}\text { CANADA } \\
\text { EGARCH }\end{array}$ & $\begin{array}{c}0.00057 \\
(0.00047) \\
{[1.215]}\end{array}$ & $\begin{array}{c}0.00115 \\
(0.00053) \\
{[2.165]^{* *}}\end{array}$ & $\begin{array}{c}8.56 \mathrm{e}-05 \\
(0.00053) \\
{[0.160]}\end{array}$ & $\begin{array}{c}0.00022 \\
(0.00060) \\
{[0.369]}\end{array}$ & $\begin{array}{c}0.00107 \\
(0.00056) \\
{[1.930]^{* * *}}\end{array}$ & $\begin{array}{c}-7.74 \mathrm{e}-05 \\
(0.00053) \\
{[-0.145]}\end{array}$ & $\begin{array}{c}0.00070 \\
(0.00054) \\
{[1.313]}\end{array}$ & $\begin{array}{c}0.00038 \\
(0.00053) \\
{[0.720]}\end{array}$ & $\begin{array}{c}0.00034 \\
(0.00049) \\
{[0.694]}\end{array}$ & $\begin{array}{c}0.00063 \\
(0.00056) \\
{[1.119]}\end{array}$ & $\begin{array}{c}0.00040 \\
(0.00060) \\
{[0.674]}\end{array}$ & $\begin{array}{c}0.00115 \\
(0.00051) \\
{[2.247]^{* *}}\end{array}$ & & & & $\begin{array}{c}0.9862 \\
(0.0035) \\
{[279.91]^{*}}\end{array}$ \\
\hline $\begin{array}{c}\text { CHILE } \\
\text { EGARCH }\end{array}$ & $\begin{array}{c}0.00085 \\
(0.00078) \\
{[1.091]}\end{array}$ & $\begin{array}{c}0.00216 \\
(0.00067) \\
{[3.219]^{*}}\end{array}$ & $\begin{array}{c}2.04 \mathrm{e}-05 \\
(0.00077) \\
{[0.026]}\end{array}$ & $\begin{array}{c}0.00043 \\
(0.00074) \\
{[0.577]}\end{array}$ & $\begin{array}{c}0.00065 \\
(0.00073) \\
{[0.885]}\end{array}$ & $\begin{array}{c}0.00135 \\
(0.00076) \\
{[1.765]^{* * *}}\end{array}$ & $\begin{array}{r}0.00 \\
(0.00 \\
{[0.7}\end{array}$ & $\begin{array}{c}0.00094 \\
(0.00060) \\
{[1.559]}\end{array}$ & $\begin{array}{c}0.00076 \\
(0.00083) \\
{[0.920]}\end{array}$ & $\begin{array}{c}0.00136 \\
(0.00068) \\
{[2.006]^{* *}}\end{array}$ & $\begin{array}{c}0.00021 \\
(0.00078) \\
{[0.276]}\end{array}$ & $\begin{array}{c}0.00094 \\
(0.00085) \\
{[1.109]}\end{array}$ & & & & \\
\hline $\begin{array}{c}\text { CHINA } \\
\text { EGARCH }\end{array}$ & $\begin{array}{c}0.00137 \\
(0.00082) \\
{[1.675]^{* * *}}\end{array}$ & $\begin{array}{c}-7.20 \mathrm{e}-05 \\
(0.00107) \\
{[-0.067]}\end{array}$ & $\begin{array}{c}0.00073 \\
(0.00070) \\
{[1.042]}\end{array}$ & $\begin{array}{c}0.00185 \\
(0.00072) \\
{[2.550]^{* *}}\end{array}$ & $\begin{array}{c}0.00111 \\
(0.00089) \\
{[1.245]}\end{array}$ & $\begin{array}{c}-0.00071 \\
(0.00078) \\
{[-0.917]}\end{array}$ & $\begin{array}{c}-0.00020 \\
(0.00073) \\
{[-0.282]}\end{array}$ & $\begin{array}{c}0.00012 \\
(0.00066) \\
{[0.193]}\end{array}$ & $\begin{array}{c}-0.00132 \\
(0.00068) \\
{[-1.95]^{* *}}\end{array}$ & $\begin{array}{c}-0.00087 \\
(0.00083) \\
{[-1.048]}\end{array}$ & $\begin{array}{c}0.00060 \\
(0.00071) \\
{[0.842]}\end{array}$ & $\begin{array}{c}-8.21 \mathrm{e}-05 \\
(0.00066) \\
{[-0.122]}\end{array}$ & & & & $\begin{array}{c}0.9726 \\
(0.0069) \\
{[140.38]^{*}}\end{array}$ \\
\hline $\begin{array}{l}\text { CROATIA } \\
\text { EGARCH }\end{array}$ & $\begin{array}{c}0.00087 \\
(0.00065) \\
{[1.348]}\end{array}$ & $\begin{array}{c}-0.00039 \\
(0.00081) \\
{[-0.484]}\end{array}$ & $\begin{array}{c}0.00114 \\
(0.00066) \\
{[1.711]^{* * *}}\end{array}$ & $\begin{array}{c}0.00246 \\
(0.00046) \\
{[5.289]^{*}}\end{array}$ & $\begin{array}{c}-0.00045 \\
(0.00072) \\
{[-0.621]}\end{array}$ & $\begin{array}{c}4.25 \mathrm{e}-05 \\
(0.00090) \\
{[0.046]}\end{array}$ & $\begin{array}{c}0.00092 \\
(0.00063) \\
{[1.455]}\end{array}$ & $\begin{array}{c}0.00066 \\
(0.00074) \\
{[0.893]}\end{array}$ & $\begin{array}{c}0.00138 \\
(0.00051) \\
{[2.731]^{*}}\end{array}$ & $\begin{array}{c}0.00280 \\
(0.00046) \\
{[6.015]^{*}}\end{array}$ & $\begin{array}{c}0.00131 \\
(0.00091) \\
{[1.438]}\end{array}$ & $\begin{array}{c}0.00037 \\
(0.00058) \\
{[0.643]}\end{array}$ & & & & $\begin{array}{c}0.9738 \\
(0.0026) \\
{[369.21]^{*}}\end{array}$ \\
\hline $\begin{array}{l}\text { DENMARK } \\
\text { GJR-GARCH }\end{array}$ & $\begin{array}{c}0.00063 \\
(0.00049) \\
{[1.300]}\end{array}$ & $\begin{array}{c}0.00040 \\
(0.00048) \\
{[0.841]}\end{array}$ & $\begin{array}{c}-0.00007 \\
(0.00049) \\
{[-0.135]}\end{array}$ & $\begin{array}{c}0.00056 \\
(0.00058) \\
{[0.959]}\end{array}$ & $\begin{array}{c}0.00077 \\
(0.00054) \\
{[1.426]}\end{array}$ & $\begin{array}{c}0.00052 \\
(0.00048) \\
{[1.084]}\end{array}$ & $\begin{array}{c}0.00099 \\
(0.00044) \\
{[2.238]^{* *}}\end{array}$ & $\begin{array}{c}0.00048 \\
(0.00048) \\
{[1.012]}\end{array}$ & $\begin{array}{c}0.00033 \\
(0.00049) \\
{[0.677]}\end{array}$ & $\begin{array}{c}0.00069 \\
(0.00048) \\
{[1.449]}\end{array}$ & $\begin{array}{c}0.00056 \\
(0.00051) \\
{[1.092]}\end{array}$ & $\begin{array}{c}0.00130 \\
(0.00050) \\
{[2.608]^{*}}\end{array}$ & $\begin{array}{c}2.53 \mathrm{e}-06 \\
(5.39 \mathrm{e}-07) \\
{[4.702]^{*}}\end{array}$ & & & $\begin{array}{c}0.8873 \\
(0.0121) \\
{[73.117]^{*}}\end{array}$ \\
\hline $\begin{array}{c}\text { EGYPT } \\
\text { EGARCH }\end{array}$ & $\begin{array}{c}0.00092 \\
(0.00030) \\
{[3.081]^{*}}\end{array}$ & $\begin{array}{c}0.00151 \\
(0.00027) \\
{[5.521]^{*}}\end{array}$ & $\begin{array}{c}0.00047 \\
(0.00059) \\
{[0.796]}\end{array}$ & $\begin{array}{c}-2.55 \mathrm{e}-05 \\
(0.00040) \\
{[-0.063]}\end{array}$ & $\begin{array}{l}-0.00126 \\
(0.00028) \\
{[-4.495]^{*}}\end{array}$ & $\begin{array}{c}0.00025 \\
(0.00051) \\
{[0.499]}\end{array}$ & $\begin{array}{c}2.88 \mathrm{e}-05 \\
(0.00058) \\
{[0.049]}\end{array}$ & $\begin{array}{c}-0.00037 \\
(0.00033) \\
{[-1.117]} \\
\end{array}$ & $\begin{array}{c}8.00 \mathrm{e}-05 \\
(0.00039) \\
{[0.203]}\end{array}$ & $\begin{array}{c}0.00067 \\
(0.00038) \\
{[1.760]^{* * *}}\end{array}$ & $\begin{array}{c}0.00014 \\
(0.00042) \\
{[0.336]}\end{array}$ & $\begin{array}{c}0.00022 \\
(0.00046) \\
{[0.486]}\end{array}$ & $\begin{array}{c}-0.4576 \\
(0.0300) \\
{[-15.221]^{*}}\end{array}$ & & & $\begin{array}{c}0.9756 \\
(0.0026) \\
{[363.03]^{*}}\end{array}$ \\
\hline $\begin{array}{l}\text { ESTONIA } \\
\text { EGARCH }\end{array}$ & $\begin{array}{c}0.00090 \\
(0.00053) \\
{[1.691]^{* * *}}\end{array}$ & $\begin{array}{c}0.00121 \\
(0.00064) \\
{[1.883]^{* * *}}\end{array}$ & $\begin{array}{c}0.00136 \\
(0.00054) \\
{[2.503]^{*}}\end{array}$ & $\begin{array}{c}0.00017 \\
(0.00051) \\
{[0.340]}\end{array}$ & $\begin{array}{c}-0.00017 \\
(0.00043) \\
{[-0.392]}\end{array}$ & $\begin{array}{c}-3.67 \mathrm{e}-05 \\
(0.00041) \\
{[-0.088]}\end{array}$ & $\begin{array}{c}0.00016 \\
(0.00039) \\
{[0.424]}\end{array}$ & $\begin{array}{c}0.00132 \\
(0.00043) \\
{[3.062]^{*}}\end{array}$ & $\begin{array}{c}0.00110 \\
(0.00045) \\
{[2.410]^{* *}}\end{array}$ & $\begin{array}{c}0.00034 \\
(0.00055) \\
{[0.616]}\end{array}$ & $\begin{array}{c}0.00110 \\
(0.00065) \\
{[1.697]^{* * *}}\end{array}$ & $\begin{array}{c}0.00149 \\
(0.00056) \\
{[2.644]^{*}}\end{array}$ & & $\begin{array}{c}0.3201 \\
(0.0411) \\
{[7.785]^{*}}\end{array}$ & $\begin{array}{c}-0.0061 \\
(0.0182) \\
{[-0.335]}\end{array}$ & $\begin{array}{c}0.9697 \\
(0.0089) \\
{[108.90]^{*}}\end{array}$ \\
\hline $\begin{array}{l}\text { FINLAND } \\
\text { EGARCH }\end{array}$ & $\begin{array}{c}0.00050 \\
(0.00085) \\
{[0.586]}\end{array}$ & $\begin{array}{c}0.00226 \\
(0.00081) \\
{[2.764]^{*}}\end{array}$ & $\begin{array}{c}0.00111 \\
(0.00073) \\
{[1.518]}\end{array}$ & $\begin{array}{c}0.00072 \\
(0.00088) \\
{[0.814]}\end{array}$ & $\begin{array}{c}0.00040 \\
(0.00097) \\
{[0.411]}\end{array}$ & $\begin{array}{c}0.00091 \\
(0.00085) \\
{[1.073]}\end{array}$ & $\begin{array}{c}0.00067 \\
(0.00075) \\
{[0.896]}\end{array}$ & $\begin{array}{c}-0.00046 \\
(0.00086) \\
{[-0.531]}\end{array}$ & $\begin{array}{c}0.00138 \\
(0.00080) \\
{[1.721]^{* * *}}\end{array}$ & $\begin{array}{c}0.00262 \\
(0.00088) \\
{[2.992]^{*}}\end{array}$ & $\begin{array}{c}0.00146 \\
(0.00090) \\
{[1.616]}\end{array}$ & $\begin{array}{c}-0.00035 \\
(0.00108) \\
{[-0.331]}\end{array}$ & $\begin{array}{c}-0.1678 \\
(0.0256) \\
{[-6.534]^{*}}\end{array}$ & $\begin{array}{c}0.1454 \\
(0.0175) \\
{[8.275]^{*}}\end{array}$ & $\begin{array}{c}-0.0382 \\
(0.0107) \\
{[-3.577]^{*}}\end{array}$ & $\begin{array}{c}0.9929 \\
(0.0023) \\
{[419.45]^{*}}\end{array}$ \\
\hline $\begin{array}{l}\text { FRANCE } \\
\text { EGARCH }\end{array}$ & $\begin{array}{c}0.00046 \\
(0.00046) \\
{[1.005]}\end{array}$ & $\begin{array}{c}0.00074 \\
(0.00048) \\
{[1.540]}\end{array}$ & $\begin{array}{c}0.00085 \\
(0.00046) \\
{[1.853]^{* * *}}\end{array}$ & $\begin{array}{c}0.00041 \\
(0.00050) \\
{[0.822]}\end{array}$ & $\begin{array}{c}-0.00015 \\
(0.00050) \\
{[-0.301]}\end{array}$ & $\begin{array}{c}-0.00053 \\
(0.00048) \\
{[-1.122]}\end{array}$ & $\begin{array}{c}0.00018 \\
(0.00047) \\
{[0.387]}\end{array}$ & $\begin{array}{c}7.62 \mathrm{e}-05 \\
(0.00048) \\
{[0.157]}\end{array}$ & $\begin{array}{c}-0.00043 \\
(0.00041) \\
{[-0.844]}\end{array}$ & $\begin{array}{c}0.00036 \\
(0.00046) \\
{[0.784]}\end{array}$ & $\begin{array}{c}0.00033 \\
(0.00049) \\
{[0.688]}\end{array}$ & $\begin{array}{c}0.00075 \\
(0.00051) \\
{[1.460]}\end{array}$ & $\begin{array}{c}-0.2287 \\
(0.0291) \\
{[-7.856]^{*}}\end{array}$ & $\begin{array}{l}0.1157 \\
(0.0123) \\
{[9.376]^{*}}\end{array}$ & $\begin{array}{c}-0.0812 \\
(0.0079) \\
{[-10.26]^{*}}\end{array}$ & $\begin{array}{c}0.9845 \\
(0.0028) \\
{[350.76]^{*}}\end{array}$ \\
\hline
\end{tabular}

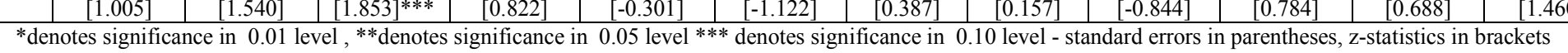


Table 3. (cont.) GARCH estimations of equation (2)

\begin{tabular}{|c|c|c|c|c|c|c|c|c|c|c|c|c|c|c|c|c|}
\hline Countries & $\beta_{1}$ & $\beta_{2}$ & $\beta_{3}$ & $\beta_{4}$ & $\beta_{5}$ & $\beta_{6}$ & $\beta_{7}$ & $\beta_{8}$ & $\beta_{9}$ & $\beta_{10}$ & $\beta_{11}$ & $\beta_{12}$ & $\omega$ & $\alpha_{0}$ & $\gamma$ & $\alpha_{1}$ \\
\hline $\begin{array}{c}\text { GERMANY } \\
\text { EGARCH }\end{array}$ & $\begin{array}{c}0.00052 \\
(0.00045) \\
{[1.147]}\end{array}$ & $\begin{array}{c}0.00117 \\
(0.00047) \\
{[2.452]^{* *}}\end{array}$ & $\begin{array}{c}-0.00021 \\
(0.00051) \\
{[-0.415]}\end{array}$ & $\begin{array}{c}0.00039 \\
(0.00051) \\
{[0.762]}\end{array}$ & $\begin{array}{c}0.00065 \\
(0.00047) \\
{[1.378]}\end{array}$ & $\begin{array}{c}-0.00012 \\
(0.00047) \\
{[-0.254]}\end{array}$ & $\begin{array}{c}0.00096 \\
(0.00046) \\
{[2.104]^{* *}}\end{array}$ & $\begin{array}{c}0.00024 \\
(0.00051) \\
{[0.481]}\end{array}$ & $\begin{array}{c}-0.00042 \\
(0.00047) \\
{[-0.890]}\end{array}$ & $\begin{array}{c}0.00072 \\
(0.00049) \\
{[1.452]}\end{array}$ & $\begin{array}{c}0.00079 \\
(0.00049) \\
{[1.608]}\end{array}$ & $\begin{array}{c}0.00160 \\
(0.00052) \\
{[3.031]^{*}}\end{array}$ & $\begin{array}{c}-0.2260 \\
(0.0284) \\
{[-7.942]^{*}}\end{array}$ & $\begin{array}{c}0.1366 \\
(0.0138) \\
{[9.891]^{*}}\end{array}$ & $\begin{array}{c}-0.0699 \\
(0.0084) \\
{[-8.313]^{*}}\end{array}$ & $\begin{array}{c}0.9866 \\
(0.0026) \\
{[378.18]^{*}}\end{array}$ \\
\hline $\begin{array}{c}\text { GREECE } \\
\text { GJR-GARCH }\end{array}$ & $\begin{array}{c}0.00122 \\
(0.00073) \\
{[1.665]^{* * *}}\end{array}$ & $\begin{array}{c}-0.00036 \\
(0.00077) \\
{[-0.461]}\end{array}$ & $\begin{array}{c}-0.00078 \\
(0.00091) \\
{[-0.854]}\end{array}$ & $\begin{array}{c}0.00178 \\
(0.00090) \\
{[1.989]^{* *}}\end{array}$ & $\begin{array}{c}0.00036 \\
(0.00078) \\
{[0.460]} \\
\end{array}$ & $\begin{array}{c}-0.00182 \\
(0.00076) \\
{[-2.375]^{* *}}\end{array}$ & $\begin{array}{c}0.00101 \\
(0.00076) \\
{[1.336]}\end{array}$ & $\begin{array}{c}0.00039 \\
(0.00077) \\
{[0.508]}\end{array}$ & $\begin{array}{c}0.00007 \\
(0.00081) \\
{[0.088]}\end{array}$ & $\begin{array}{c}0.00082 \\
(0.00082) \\
{[0.990]}\end{array}$ & $\begin{array}{c}0.00118 \\
(0.00076) \\
{[1.551]}\end{array}$ & $\begin{array}{c}0.00099 \\
(0.00082) \\
{[1.205]}\end{array}$ & $\begin{array}{c}7.16 \mathrm{e}-06 \\
(1.49 \mathrm{e}-06) \\
{[4.792]^{*}}\end{array}$ & $\begin{array}{c}0.0940 \\
(0.0165) \\
{[5.682]^{*}}\end{array}$ & $\begin{array}{c}0.1014 \\
(0.0231) \\
{[4.389]^{*}}\end{array}$ & $\begin{array}{c}0.8364 \\
(0.0156) \\
{[53.709]^{*}}\end{array}$ \\
\hline $\begin{array}{c}\text { HONG } \\
\text { KONG } \\
\text { EGARCH }\end{array}$ & $\begin{array}{c}0.00112 \\
(0.00049) \\
{[2.271]^{* *}}\end{array}$ & $\begin{array}{c}0.00156 \\
(0.00055) \\
{[2.820]^{*}}\end{array}$ & $\begin{array}{c}-0.00054 \\
(0.00051) \\
{[-1.055]}\end{array}$ & $\begin{array}{c}0.00070 \\
(0.00050) \\
{[1.269]}\end{array}$ & $\begin{array}{c}0.00109 \\
(0.00050) \\
{[2.197]^{*}}\end{array}$ & $\begin{array}{c}0.00027 \\
(0.00050) \\
{[0.545]}\end{array}$ & $\begin{array}{c}0.00117 \\
(0.00046) \\
{[2.539]^{* *}}\end{array}$ & $\begin{array}{c}7.30 \mathrm{e}-05 \\
(0.00047) \\
{[0.155]}\end{array}$ & $\begin{array}{c}0.00049 \\
(0.00050) \\
{[0.983]}\end{array}$ & $\begin{array}{c}0.00115 \\
(0.00051) \\
{[2.229]^{* *}}\end{array}$ & $\begin{array}{c}0.00095 \\
(0.00050) \\
{[1.894]^{* * *}}\end{array}$ & $\begin{array}{c}0.00100 \\
(0.00053) \\
{[1.864]^{* * *}}\end{array}$ & $\begin{array}{c}-0.2951 \\
(0.0324) \\
{[-9.094]^{*}}\end{array}$ & $\begin{array}{c}0.1613 \\
(0.0138) \\
{[11.610]^{*}}\end{array}$ & $\begin{array}{c}-0.0584 \\
(0.0083) \\
{[-7.045]^{*}}\end{array}$ & $\begin{array}{c}0.9796 \\
(0.0031) \\
{[308.02]^{*}}\end{array}$ \\
\hline $\begin{array}{c}\text { INDIA } \\
\text { GJR-GARCH }\end{array}$ & $\begin{array}{c}-0.00019 \\
(0.00079) \\
{[-0.239]}\end{array}$ & $\begin{array}{c}0.00148 \\
(0.00079) \\
{[1.857]^{* * *}}\end{array}$ & $\begin{array}{c}-0.00067 \\
(0.00091) \\
{[-0.736]}\end{array}$ & $\begin{array}{c}-0.00021 \\
(0.00094) \\
{[-0.223]}\end{array}$ & $\begin{array}{c}0.00134 \\
(0.00092) \\
{[1.456]}\end{array}$ & $\begin{array}{c}0.00095 \\
(0.00087) \\
{[1.094]}\end{array}$ & $\begin{array}{c}0.00110 \\
(0.00079) \\
{[1.397]}\end{array}$ & $\begin{array}{c}0.00093 \\
(0.00078) \\
{[1.201]}\end{array}$ & $\begin{array}{c}0.00153 \\
(0.00080) \\
{[1.929]^{* * *}}\end{array}$ & $\begin{array}{c}-0.00003 \\
(0.00079) \\
{[-0.038]}\end{array}$ & $\begin{array}{c}0.00232 \\
(0.00085) \\
{[2.723]^{*}}\end{array}$ & $\begin{array}{c}0.00260 \\
(0.00081) \\
{[3.203]^{*}}\end{array}$ & $\begin{array}{c}1.19 \mathrm{e}-05 \\
(2.15 \mathrm{e}-06) \\
{[5.547]^{*}}\end{array}$ & $\begin{array}{c}0.0536 \\
(0.0184) \\
{[2.922]^{*}}\end{array}$ & $\begin{array}{c}0.1731 \\
(0.0280) \\
{[6.192]^{*}}\end{array}$ & $\begin{array}{c}0.8167 \\
(0.0195) \\
{[41.956]^{*}}\end{array}$ \\
\hline $\begin{array}{l}\text { INDONESIA } \\
\text { GJR-GARCH }\end{array}$ & $\begin{array}{c}0.00143 \\
(0.00083) \\
{[1.715]^{* * *}}\end{array}$ & $\begin{array}{c}-0.00019 \\
(0.00085) \\
{[-0.217]}\end{array}$ & $\begin{array}{c}0.00066 \\
(0.00082) \\
{[0.810]}\end{array}$ & $\begin{array}{c}0.00163 \\
(0.00087) \\
{[1.876]^{* * *}}\end{array}$ & $\begin{array}{c}0.00225 \\
(0.00089) \\
{[2.541]^{* *}}\end{array}$ & $\begin{array}{c}0.00073 \\
(0.00082) \\
{[0.883]}\end{array}$ & $\begin{array}{c}0.00064 \\
(0.00072) \\
{[0.880]}\end{array}$ & $\begin{array}{c}-0.00158 \\
(0.00084) \\
{[-1.86]^{* * *}}\end{array}$ & $\begin{array}{c}0.00100 \\
(0.00091) \\
{[1.097]}\end{array}$ & $\begin{array}{c}0.00022 \\
(0.00086) \\
{[0.252]}\end{array}$ & $\begin{array}{c}0.00179 \\
(0.00081) \\
{[2.222]^{* *}}\end{array}$ & $\begin{array}{c}0.00246 \\
(0.00086) \\
{[2.846]^{*}}\end{array}$ & $\begin{array}{c}1.92 \mathrm{e}-05 \\
(3.41 \mathrm{e}-06) \\
{[5.640]^{*}}\end{array}$ & $\begin{array}{c}0.0945 \\
(0.0205) \\
{[4.607]^{*}}\end{array}$ & $\begin{array}{c}0.1635 \\
(0.0351) \\
{[4.661]^{*}}\end{array}$ & $\begin{array}{c}0.7718 \\
(0.0246) \\
{[31.359]^{*}}\end{array}$ \\
\hline $\begin{array}{l}\text { IRELAND } \\
\text { EGARCH }\end{array}$ & $\begin{array}{c}2.67 \mathrm{e}-05 \\
(0.00021) \\
{[0.128]}\end{array}$ & $\begin{array}{c}1.42 \mathrm{e}-05 \\
(0.00023) \\
{[0.059]}\end{array}$ & $\begin{array}{c}-2.85 \mathrm{e}-07 \\
(0.00025) \\
{[-0.011]}\end{array}$ & $\begin{array}{c}1.82 \mathrm{e}-05 \\
(0.00025) \\
{[0.071]}\end{array}$ & $\begin{array}{c}9.88 \mathrm{e}-07 \\
(0.00026) \\
{[0.003]}\end{array}$ & $\begin{array}{c}1.06 \mathrm{e}-05 \\
(0.00026) \\
{[-0.004]}\end{array}$ & $\begin{array}{c}0.00032 \\
(0.00022) \\
{[1.420]}\end{array}$ & $\begin{array}{c}-5.36 \mathrm{e}-07 \\
(0.00025) \\
{[-0.002]}\end{array}$ & $\begin{array}{c}-1.51 \mathrm{e}-05 \\
(0.00025) \\
{[-0.059]}\end{array}$ & $\begin{array}{c}1.78 \mathrm{e}-05 \\
(0.00025) \\
{[0.071]}\end{array}$ & $\begin{array}{c}1.01 \mathrm{e}-05 \\
(0.00026) \\
{[0.037]}\end{array}$ & $\begin{array}{c}0.00057 \\
(0.00024) \\
{[2.397]^{* *}}\end{array}$ & $\begin{array}{c}-0.0605 \\
(0.0079) \\
{[-7.652]^{*}}\end{array}$ & & & $\begin{array}{c}0.9978 \\
(0.0094) \\
{[1061.1]^{*}}\end{array}$ \\
\hline $\begin{array}{c}\text { ISRAEL } \\
\text { EGARCH }\end{array}$ & $\begin{array}{c}-0.00073 \\
(0.00086) \\
{[-0.846]}\end{array}$ & $\begin{array}{c}0.00062 \\
(0.00081) \\
{[0.765]}\end{array}$ & $\begin{array}{c}0.00105 \\
(0.00080) \\
{[1.316]}\end{array}$ & $\begin{array}{c}0.00204 \\
(0.00094) \\
{[2.159]^{* *}}\end{array}$ & $\begin{array}{c}0.00126 \\
(0.00088) \\
{[1.428]}\end{array}$ & $\begin{array}{c}-0.00068 \\
(0.00086) \\
{[-0.799]}\end{array}$ & $\begin{array}{c}-7.29 \mathrm{e}-05 \\
(0.00074) \\
{[-0.097]}\end{array}$ & $\begin{array}{c}-0.00097 \\
(0.00077) \\
{[-1.248]}\end{array}$ & $\begin{array}{c}-0.00016 \\
(0.00078) \\
{[-0.208]}\end{array}$ & $\begin{array}{c}0.00201 \\
(0.00085) \\
{[2.365]^{* *}}\end{array}$ & $\begin{array}{c}0.00187 \\
(0.00089) \\
{[2.103]^{* *}}\end{array}$ & $\begin{array}{c}0.00185 \\
(0.00079) \\
{[2.331]^{* *}}\end{array}$ & & $\begin{array}{c}0.1909 \\
(0.0287) \\
{[6.634]^{*}}\end{array}$ & $\begin{array}{c}-0.1133 \\
(0.0188) \\
{[-6.014]^{*}}\end{array}$ & $\begin{array}{c}0.9222 \\
(0.0164) \\
{[56.052]^{*}}\end{array}$ \\
\hline $\begin{array}{c}\text { ITALY } \\
\text { EGARCH }\end{array}$ & $\begin{array}{c}0.00086 \\
(0.00039) \\
{[2.155]^{* *}}\end{array}$ & $\begin{array}{c}0.00121 \\
(0.00051) \\
{[2.369]^{* *}}\end{array}$ & $\begin{array}{c}0.00017 \\
(0.00056) \\
{[0.312]}\end{array}$ & $\begin{array}{c}0.00052 \\
(0.00054) \\
{[0.973]}\end{array}$ & $\begin{array}{c}-0.00029 \\
(0.00057) \\
{[-0.514]}\end{array}$ & $\begin{array}{c}-0.00056 \\
(0.00053) \\
{[-1.052]}\end{array}$ & $\begin{array}{c}-0.00033 \\
(0.00053) \\
{[-0.620]}\end{array}$ & $\begin{array}{c}-4.50 \mathrm{e}-05 \\
(0.00049) \\
{[-0.092]}\end{array}$ & $\begin{array}{c}0.00036 \\
(0.00055) \\
{[0.819]}\end{array}$ & $\begin{array}{c}0.00052 \\
(0.00051) \\
{[1.028]}\end{array}$ & $\begin{array}{c}0.00064 \\
(0.00046) \\
{[1.397]}\end{array}$ & $\begin{array}{c}0.00059 \\
(0.00045) \\
{[1.291]}\end{array}$ & $\begin{array}{c}-0.1991 \\
(0.0290) \\
{[-6.848]^{*}}\end{array}$ & $\begin{array}{c}0.0846 \\
(0.0169) \\
{[4.985]^{*}}\end{array}$ & $\begin{array}{c}-0.1124 \\
(0.0103) \\
{[-10.90]^{*}}\end{array}$ & $\begin{array}{c}0.9861 \\
(0.0025) \\
{[395.62]^{*}}\end{array}$ \\
\hline $\begin{array}{c}\text { JAPAN } \\
\text { EGARCH }\end{array}$ & $\begin{array}{c}0.00030 \\
(0.00035) \\
{[0.844]}\end{array}$ & $\begin{array}{c}0.00081 \\
(0.00039) \\
{[2.060]^{* *}}\end{array}$ & $\begin{array}{c}0.00165 \\
(0.00035) \\
{[4.695]^{*}}\end{array}$ & $\begin{array}{c}0.00050 \\
(0.00044) \\
{[1.124]}\end{array}$ & $\begin{array}{c}5.46 \mathrm{e}-05 \\
(0.00044) \\
{[0.123]}\end{array}$ & $\begin{array}{c}9.32 \mathrm{e}-07 \\
(0.00039) \\
{[0.002]}\end{array}$ & $\begin{array}{c}-0.000101 \\
(0.00039) \\
{[-0.255]}\end{array}$ & $\begin{array}{c}2.23 \mathrm{e}-05 \\
(0.00040) \\
{[0.055]}\end{array}$ & $\begin{array}{c}4.49 \mathrm{e}-05 \\
(0.00037) \\
{[0.119]}\end{array}$ & $\begin{array}{c}3.68 \mathrm{e}-06 \\
(0.00039) \\
{[0.009]}\end{array}$ & $\begin{array}{c}0.00045 \\
(0.00040) \\
{[1.114]}\end{array}$ & $\begin{array}{c}0.00079 \\
(0.00041) \\
{[1.936]^{* * *}}\end{array}$ & $\begin{array}{c}-0.3215 \\
(0.0298) \\
{[-10.76]^{*}}\end{array}$ & $\begin{array}{c}0.1681 \\
(0.0133) \\
{[12.554]^{*}}\end{array}$ & $\begin{array}{c}-0.0918 \\
(0.0085) \\
{[-10.772]^{*}}\end{array}$ & $\begin{array}{c}0.9784 \\
(0.0028) \\
{[344.85]^{*}}\end{array}$ \\
\hline $\begin{array}{l}\text { JORDAN } \\
\text { EGARCH }\end{array}$ & $\begin{array}{c}0.00026 \\
(0.00031) \\
{[0.841]}\end{array}$ & $\begin{array}{c}0.00015 \\
(0.00031) \\
{[0.495]}\end{array}$ & $\begin{array}{c}-0.00074 \\
(0.00027) \\
{[-2.681]^{*}}\end{array}$ & $\begin{array}{c}-0.00031 \\
(0.00033) \\
{[-0.949]}\end{array}$ & $\begin{array}{c}0.00057 \\
(0.00033) \\
{[1.710]}\end{array}$ & $\begin{array}{c}-0.00018 \\
(0.00031) \\
{[-0.581]}\end{array}$ & $\begin{array}{c}-0.00075 \\
(0.00033) \\
{[-2.248]^{* *}}\end{array}$ & $\begin{array}{c}-1.86 \mathrm{e}-05 \\
(0.00028) \\
{[-0.065]}\end{array}$ & $\begin{array}{c}0.00027 \\
(0.00029) \\
{[0.944]}\end{array}$ & $\begin{array}{c}-0.00013 \\
(0.00030) \\
{[-0.428]}\end{array}$ & $\begin{array}{c}0.00011 \\
(0.00030) \\
{[0.372]}\end{array}$ & $\begin{array}{c}0.00012 \\
(0.00029) \\
{[0.415]}\end{array}$ & $\begin{array}{c}-0.9031 \\
(0.088) \\
{[-10.26]^{*}}\end{array}$ & $\begin{array}{c}0.4637 \\
(0.0299) \\
{[15.478]^{*}}\end{array}$ & $\begin{array}{c}0.0128 \\
(0.0151) \\
{[0.852]}\end{array}$ & $\begin{array}{c}0.9412 \\
(0.0080) \\
{[117.10]^{*}}\end{array}$ \\
\hline $\begin{array}{l}\text { KUWAIT } \\
\text { EGARCH }\end{array}$ & $\begin{array}{c}0.00092 \\
(0.00042) \\
{[2.208 * *}\end{array}$ & $\begin{array}{c}0.00073 \\
(0.00045) \\
{[1.638]}\end{array}$ & $\begin{array}{c}0.00180 \\
(0.00051) \\
{[3.515]^{*}}\end{array}$ & $\begin{array}{c}0.00208 \\
(0.00056) \\
{[3.672]^{*}}\end{array}$ & $\begin{array}{c}0.00090 \\
(0.00057) \\
{[1.563]}\end{array}$ & $\begin{array}{c}0.00071 \\
(0.00048) \\
{[1.481]}\end{array}$ & $\begin{array}{c}0.00082 \\
(0.00052) \\
{[1.569]}\end{array}$ & $\begin{array}{c}0.00078 \\
(0.00049) \\
{[1.590]}\end{array}$ & $\begin{array}{c}0.00067 \\
(0.00044) \\
{[1.505]}\end{array}$ & $\begin{array}{c}0.00104 \\
(0.00046) \\
{[2.254]^{* *}}\end{array}$ & $\begin{array}{c}0.00098 \\
(0.00050) \\
{[1.959]^{* *}}\end{array}$ & $\begin{array}{c}0.00117 \\
(0.00043) \\
{[2.742]^{*}}\end{array}$ & $\begin{array}{c}-1.0117 \\
(0.1430) \\
{[-7.072]^{*}}\end{array}$ & $\begin{array}{c}0.3711 \\
(0.0397) \\
{[9.327]^{*}}\end{array}$ & $\begin{array}{c}-0.1072 \\
(0.0213) \\
{[-5.032]^{*}}\end{array}$ & $\begin{array}{c}0.9246 \\
(0.0136) \\
{[67.797]^{*}}\end{array}$ \\
\hline $\begin{array}{l}\text { LATVIA } \\
\text { EGARCH }\end{array}$ & $\begin{array}{c}0.00070 \\
(0.00048) \\
{[1.456]}\end{array}$ & $\begin{array}{c}-0.00031 \\
(0.00053) \\
{[-0.578]}\end{array}$ & $\begin{array}{c}0.00084 \\
(0.00055) \\
{[1.548]}\end{array}$ & $\begin{array}{c}0.00098 \\
(0.00050) \\
{[1.933]^{* * *}}\end{array}$ & $\begin{array}{c}-0.00077 \\
(0.00052) \\
{[-1.473]}\end{array}$ & $\begin{array}{c}0.00054 \\
(0.00046) \\
{[1.179]}\end{array}$ & $\begin{array}{c}0.00114 \\
(0.00055) \\
{[2.086]^{* *}}\end{array}$ & $\begin{array}{c}0.00064 \\
(0.00059) \\
{[1.092]}\end{array}$ & $\begin{array}{c}0.00078 \\
(0.00055) \\
{[1.434]}\end{array}$ & $\begin{array}{c}3.37 \mathrm{e}-05 \\
(0.00054) \\
{[0.062]}\end{array}$ & $\begin{array}{c}0.00070 \\
(0.00059) \\
{[1.194]}\end{array}$ & $\begin{array}{c}0.00101 \\
(0.00050) \\
{[2.009]^{* *}}\end{array}$ & $\begin{array}{c}-1.0211 \\
(0.1337) \\
{[-7.635]^{*}}\end{array}$ & $\begin{array}{c}0.4843 \\
(0.0485) \\
{[9.979]^{*}}\end{array}$ & $\begin{array}{c}-0.0324 \\
(0.0255) \\
{[-1.271]}\end{array}$ & $\begin{array}{c}0.9209 \\
(0.0134) \\
{[68.376]^{*}}\end{array}$ \\
\hline $\begin{array}{l}\text { LITHUANIA } \\
\text { GJR-GARCH }\end{array}$ & $\begin{array}{c}0.00097 \\
(0.00048) \\
{[2.019]^{* *}} \\
\end{array}$ & $\begin{array}{c}0.00087 \\
(0.00056) \\
{[1.544]} \\
\end{array}$ & $\begin{array}{c}0.00161 \\
(0.00050) \\
{[3.223]^{*}} \\
\end{array}$ & $\begin{array}{c}0.00002 \\
(0.00053) \\
{[0.037]}\end{array}$ & $\begin{array}{c}-0.00046 \\
(0.00052) \\
{[-0.894]}\end{array}$ & $\begin{array}{c}-0.00027 \\
(0.00052) \\
{[-0.524]}\end{array}$ & $\begin{array}{c}0.00015 \\
(0.00050) \\
{[0.300]}\end{array}$ & $\begin{array}{c}0.00094 \\
(0.00049) \\
{[1.923]^{* * *}}\end{array}$ & $\begin{array}{c}0.00174 \\
(0.00054) \\
{[3.242]^{*}} \\
\end{array}$ & $\begin{array}{c}0.00053 \\
(0.00052) \\
{[1.020]}\end{array}$ & $\begin{array}{c}0.00136 \\
(0.00055) \\
{[2.479]^{* *}}\end{array}$ & $\begin{array}{c}0.00094 \\
(0.00053) \\
{[1.785]^{* * *}}\end{array}$ & $\begin{array}{c}1.56 \mathrm{e}-05 \\
(2.83 \mathrm{e}-06) \\
{[5.513]^{*}}\end{array}$ & $\begin{array}{c}0.2184 \\
(0.0460) \\
{[4.748]^{*}}\end{array}$ & $\begin{array}{c}0.1643 \\
(0.0633) \\
{[2.595]^{*}}\end{array}$ & $\begin{array}{c}0.5666 \\
(0.0512) \\
{[11.072]^{*}}\end{array}$ \\
\hline $\begin{array}{c}\text { LUXEMBURG } \\
\text { GARCH }\end{array}$ & $\begin{array}{c}-0.00124 \\
(0.00045) \\
{[-2.744]^{*}}\end{array}$ & $\begin{array}{c}-0.00086 \\
(0.00068) \\
{[-1.258]}\end{array}$ & $\begin{array}{c}-0.00023 \\
(0.00061) \\
{[-0.379]}\end{array}$ & $\begin{array}{c}0.0003 \\
(0.00055) \\
{[0547]}\end{array}$ & $\begin{array}{c}9.20 \mathrm{e}-05 \\
(0.00055) \\
{[0.167]}\end{array}$ & $\begin{array}{c}0.00017 \\
(0.00057) \\
{[0.309]}\end{array}$ & $\begin{array}{c}-0.0005 \\
(0.00039) \\
{[-1.281]}\end{array}$ & $\begin{array}{c}-7.54 \mathrm{e}-05 \\
(0.00075) \\
{[-0.001]}\end{array}$ & $\begin{array}{c}6.98 \mathrm{e}-05 \\
(0.00047) \\
{[0.148]}\end{array}$ & $\begin{array}{c}-2.83 e-05 \\
(0.00044) \\
{[-0.063]}\end{array}$ & $\begin{array}{c}0.00283 \\
(0.000108) \\
{[26.256]^{*}}\end{array}$ & $\begin{array}{c}0.00305 \\
(0.00043) \\
{[7.117]^{*}}\end{array}$ & $\begin{array}{c}4.92 \mathrm{e}-08 \\
(7.42 \mathrm{e}-09) \\
{[6.634]^{*}}\end{array}$ & $\begin{array}{c}0.1620 \\
(0.0007) \\
{[23.186]^{*}}\end{array}$ & & $\begin{array}{c}0.8771 \\
(0.0035) \\
{[244.61]^{*}}\end{array}$ \\
\hline
\end{tabular}

*denotes significance in 0.01 level, ${ }^{* *}$ denotes significance in 0.05 level *** denotes significance in 0.10 level - standard errors in parentheses, $z$-statistics in brackets 
Table 3. (cont.) GARCH estimations of equation (2)

\begin{tabular}{|c|c|c|c|c|c|c|c|c|c|c|c|c|c|c|c|c|}
\hline Countries & $\beta_{1}$ & $\boldsymbol{\beta}_{2}$ & $\beta_{3}$ & $\beta_{4}$ & $\beta_{5}$ & $\beta_{6}$ & $\beta_{7}$ & $\boldsymbol{\beta}_{8}$ & $\boldsymbol{\beta}_{9}$ & $\boldsymbol{\beta}_{\mathbf{1 0}}$ & $\boldsymbol{\beta}_{11}$ & $\beta_{12}$ & $\omega$ & $\alpha_{0}$ & $\gamma$ & $\alpha_{1}$ \\
\hline $\begin{array}{l}\text { MALAYSIA } \\
\text { EGARCH }\end{array}$ & $\begin{array}{c}0.00098 \\
(0.00045) \\
{[2.172]^{* *}}\end{array}$ & $\begin{array}{c}0.00038 \\
(0.00054) \\
{[0.702]}\end{array}$ & $\begin{array}{c}-0.00055 \\
(0.00042) \\
{[-1.313]}\end{array}$ & $\begin{array}{c}0.00050 \\
(0.00045) \\
{[1.124]}\end{array}$ & $\begin{array}{c}-0.00050 \\
(0.00047) \\
{[-1.077]}\end{array}$ & $\begin{array}{c}9.36 \mathrm{e}-06 \\
(0.00045) \\
{[0.020]}\end{array}$ & $\begin{array}{c}0.00027 \\
(0.00044) \\
{[0.615]}\end{array}$ & $\begin{array}{c}-0.00015 \\
(0.00039) \\
{[-0.395]}\end{array}$ & $\begin{array}{c}-0.00020 \\
(0.00039) \\
{[-0.505]}\end{array}$ & $\begin{array}{c}0.00024 \\
(0.00036) \\
{[0.699]}\end{array}$ & $\begin{array}{c}0.00027 \\
(0.00040) \\
{[0.674]}\end{array}$ & $\begin{array}{c}0.00091 \\
(0.00046) \\
{[1.965]^{* *}}\end{array}$ & $\begin{array}{c}-0.3382 \\
(0.0374) \\
{[-9.027]^{*}}\end{array}$ & $\begin{array}{c}0.2373 \\
(0.0202) \\
{[11.743]^{*}}\end{array}$ & $\begin{array}{c}-0.0580 \\
(0.0105) \\
{[-5.509]^{*}}\end{array}$ & $\begin{array}{c}0.9821 \\
(0.0033) \\
{[296.99]^{*}}\end{array}$ \\
\hline $\begin{array}{l}\text { MEXICO } \\
\text { EGARCH }\end{array}$ & $\begin{array}{c}0.00141 \\
(0.00058) \\
{[2.409]^{* *}}\end{array}$ & $\begin{array}{c}0.00085 \\
(0.00061) \\
{[1.373]}\end{array}$ & $\begin{array}{c}0.00125 \\
(0.00065) \\
{[1.907]^{* * *}}\end{array}$ & $\begin{array}{c}0.00011 \\
(0.00062) \\
{[0.181]}\end{array}$ & $\begin{array}{c}0.00043 \\
(0.00062) \\
{[0.700]}\end{array}$ & $\begin{array}{c}3.45 \mathrm{e}-05 \\
(0.00062) \\
{[0.055]}\end{array}$ & $\begin{array}{c}0.00042 \\
(0.00054) \\
{[0.783]}\end{array}$ & $\begin{array}{c}0.00016 \\
(0.00061) \\
{[0.262]}\end{array}$ & $\begin{array}{c}0.00073 \\
(0.00062) \\
{[1.174]}\end{array}$ & $\begin{array}{c}0.00138 \\
(0.00062) \\
{[2.209]^{* *}}\end{array}$ & $\begin{array}{c}0.00172 \\
(0.00065) \\
{[2.658]}\end{array}$ & $\begin{array}{c}0.00201 \\
(0.00060) \\
{[3.329]^{*}}\end{array}$ & $\begin{array}{c}-0.4344 \\
(0.0483) \\
{[-8.978]^{*}}\end{array}$ & $\begin{array}{c}0.1921 \\
(0.0180) \\
{[10.667]^{*}}\end{array}$ & $\begin{array}{c}-0.1041 \\
(0.0102) \\
{[-10.191]^{*}}\end{array}$ & $\begin{array}{c}0.9663 \\
(0.0049) \\
{[194.62]^{*}}\end{array}$ \\
\hline $\begin{array}{c}\text { NETHERLANDS } \\
\text { GJR-GARCH }\end{array}$ & $\begin{array}{c}0.00034 \\
(0.00043) \\
{[0.777]}\end{array}$ & $\begin{array}{c}0.00090 \\
(0.00047) \\
{[1.939]^{* * *}}\end{array}$ & $\begin{array}{c}0.00024 \\
(0.00046) \\
{[0.525]}\end{array}$ & $\begin{array}{c}0.00062 \\
(0.00051) \\
{[1.210]}\end{array}$ & $\begin{array}{c}0.00002 \\
(0.00048) \\
{[0.044]}\end{array}$ & $\begin{array}{c}0.00045 \\
(0.00048) \\
{[0.944]}\end{array}$ & $\begin{array}{c}0.00097 \\
(0.00046) \\
{[2.142]^{* *}}\end{array}$ & $\begin{array}{c}0.00032 \\
(0.00048) \\
{[0.655]}\end{array}$ & $\begin{array}{c}-0.00016 \\
(0.00048) \\
{[-0.343]}\end{array}$ & $\begin{array}{c}0.00031 \\
(0.00045) \\
{[0.699]}\end{array}$ & $\begin{array}{c}0.00069 \\
(0.00049) \\
{[1.421]}\end{array}$ & $\begin{array}{c}0.00128 \\
(0.00046) \\
{[2.785]^{*}}\end{array}$ & $\begin{array}{c}1.55 \mathrm{e}-06 \\
(2.82 \mathrm{e}-07) \\
{[5.477]^{*}}\end{array}$ & $\begin{array}{c}0.0243 \\
(0.0098) \\
{[2.477]^{* *}}\end{array}$ & $\begin{array}{c}0.1084 \\
(0.0134) \\
{[8.116]^{*}}\end{array}$ & $\begin{array}{c}0.9095 \\
(0.0083) \\
{[109.07]^{*}}\end{array}$ \\
\hline $\begin{array}{l}\text { NEW ZEALAND } \\
\text { EGARCH }\end{array}$ & $\begin{array}{l}-0.0002 \\
(0.0006) \\
{[-0.282]}\end{array}$ & $\begin{array}{c}0.0007 \\
(0.0005) \\
{[1.254]}\end{array}$ & $\begin{array}{c}0.0010 \\
(0.0006) \\
{[1.558]}\end{array}$ & $\begin{array}{c}0.0007 \\
(0.0008) \\
{[0.856]}\end{array}$ & $\begin{array}{c}0.0000 \\
(0.0004) \\
{[-0.058]}\end{array}$ & $\begin{array}{c}0.0002 \\
(0.0005) \\
{[0.437]}\end{array}$ & $\begin{array}{c}0.0005 \\
(0.0006) \\
{[0.770]}\end{array}$ & $\begin{array}{c}-0.0010 \\
(0.0005) \\
{[-1.94]^{* * *}}\end{array}$ & $\begin{array}{c}0.0011 \\
(0.0006) \\
{[1.959]^{* * *}}\end{array}$ & $\begin{array}{c}-0.0001 \\
(0.0006) \\
{[-0.088]}\end{array}$ & $\begin{array}{c}0.0005 \\
(0.0005) \\
{[1.006]}\end{array}$ & $\begin{array}{c}0.0011 \\
(0.0006) \\
{[1.820]^{* * *}}\end{array}$ & & & & $\begin{array}{c}0.9824 \\
(0.0062) \\
{[158.16]^{*}}\end{array}$ \\
\hline $\begin{array}{c}\text { NORWAY } \\
\text { GJR-GARCH }\end{array}$ & $\begin{array}{c}0.00081 \\
(0.00081) \\
{[1.004]} \\
\end{array}$ & $\begin{array}{c}0.00114 \\
(0.00083) \\
{[1.370]}\end{array}$ & $\begin{array}{c}0.00047 \\
(0.00077) \\
{[0.605]}\end{array}$ & $\begin{array}{c}0.00161 \\
(0.00075) \\
{[2.154]^{* *}}\end{array}$ & $\begin{array}{c}0.00165 \\
(0.00082) \\
{[2.011]^{* *}} \\
\end{array}$ & $\begin{array}{c}0.00095 \\
(0.00084) \\
{[1.133]} \\
\end{array}$ & $\begin{array}{c}0.00011 \\
(0.00077) \\
{[0.148]}\end{array}$ & $\begin{array}{c}-0.00005 \\
(0.00080) \\
{[-0.059]}\end{array}$ & $\begin{array}{c}0.00012 \\
(0.00088) \\
{[0.135]}\end{array}$ & $\begin{array}{c}0.00197 \\
(0.00084) \\
{[2.347]^{* *}} \\
\end{array}$ & $\begin{array}{c}0.00106 \\
(0.00086) \\
{[1.228]}\end{array}$ & $\begin{array}{c}0.00121 \\
(0.00086) \\
{[1.405]}\end{array}$ & $\begin{array}{c}1.31 \mathrm{e}-05 \\
(2.45 \mathrm{e}-06) \\
{[5.361]^{*}}\end{array}$ & & $\begin{array}{c}0.2099 \\
(0.0359) \\
{[5.852]^{*}}\end{array}$ & $\begin{array}{c}0.7819 \\
(0.0294) \\
{[26.617]^{*}}\end{array}$ \\
\hline $\begin{array}{l}\text { PAKISTAN } \\
\text { GJR-GARCH }\end{array}$ & $\begin{array}{c}0.00342 \\
(0.00081) \\
{[4.230]^{*}}\end{array}$ & $\begin{array}{c}0.00180 \\
(0.00082) \\
{[2.190]^{* *}}\end{array}$ & $\begin{array}{c}0.00107 \\
(0.00088) \\
{[1.217]}\end{array}$ & $\begin{array}{c}0.00226 \\
(0.00072) \\
{[3.124]^{*}}\end{array}$ & $\begin{array}{c}-0.00032 \\
(0.00087) \\
{[-0.364]}\end{array}$ & $\begin{array}{c}0.00175 \\
(0.00098) \\
{[1.788]^{* * *}}\end{array}$ & $\begin{array}{c}0.00044 \\
(0.00070) \\
{[0.638]}\end{array}$ & $\begin{array}{c}0.00076 \\
(0.00074) \\
{[1.031]}\end{array}$ & $\begin{array}{c}0.00107 \\
(0.00075) \\
{[1.417]}\end{array}$ & $\begin{array}{c}0.00308 \\
(0.00070) \\
{[4.381]^{*}}\end{array}$ & $\begin{array}{c}0.00108 \\
(0.00088) \\
{[1.230]}\end{array}$ & $\begin{array}{c}0.00308 \\
(0.00078) \\
{[3.940]^{*}}\end{array}$ & $\begin{array}{c}1.24 \mathrm{e}-05 \\
(2.40 \mathrm{e}-06) \\
{[5.157]^{*}}\end{array}$ & $\begin{array}{c}0.1801 \\
(0.0323) \\
{[5.584]^{*}}\end{array}$ & $\begin{array}{c}0.0860 \\
(0.0388) \\
{[2.215]^{* *}}\end{array}$ & $\begin{array}{c}0.7526 \\
(0.0250) \\
{[30.118]}\end{array}$ \\
\hline $\begin{array}{c}\text { PERU } \\
\text { GJR-GARCH }\end{array}$ & $\begin{array}{c}0.00246 \\
(0.00064) \\
{[3.860]^{*}}\end{array}$ & $\begin{array}{c}0.00111 \\
(0.00060) \\
{[1.847]^{* * *}}\end{array}$ & $\begin{array}{c}0.00079 \\
(0.00053) \\
{[1.497]}\end{array}$ & $\begin{array}{c}-0.00018 \\
(0.00068) \\
{[-0.258]}\end{array}$ & $\begin{array}{c}0.00051 \\
(0.00058) \\
{[0.882]}\end{array}$ & $\begin{array}{c}-0.00075 \\
(0.00059) \\
{[-1.264]}\end{array}$ & $\begin{array}{c}0.00029 \\
(0.00053) \\
{[0.544]}\end{array}$ & $\begin{array}{c}0.00061 \\
(0.00058) \\
{[1.045]}\end{array}$ & $\begin{array}{c}0.00193 \\
(0.00060) \\
{[3.223]^{*}}\end{array}$ & $\begin{array}{c}0.00081 \\
(0.00058) \\
{[1.399]}\end{array}$ & $\begin{array}{c}0.00103 \\
(0.00056) \\
{[1.846]^{* * *}}\end{array}$ & $\begin{array}{c}0.00063 \\
(0.00063) \\
{[0.997]}\end{array}$ & $\begin{array}{c}8.13 \mathrm{e}-06 \\
(1.43 \mathrm{e}-06) \\
{[5.691]^{*}}\end{array}$ & $\begin{array}{c}0.2203 \\
(0.0331) \\
{[6.665]^{*}}\end{array}$ & $\begin{array}{c}0.0631 \\
(0.0375) \\
{[1.682]^{* * *}}\end{array}$ & $\begin{array}{c}0.7096 \\
(0.0266) \\
{[26.652]^{*}}\end{array}$ \\
\hline $\begin{array}{l}\text { PHILLIPINE } \\
\text { GJR-GARCH }\end{array}$ & $\begin{array}{c}0.00160 \\
(0.00081) \\
{[1.986]^{* *}}\end{array}$ & $\begin{array}{c}-0.00053 \\
(0.00086) \\
{[-0.612]}\end{array}$ & $\begin{array}{c}-0.00035 \\
(0.00079) \\
{[-0.438]}\end{array}$ & $\begin{array}{c}0.00017 \\
(0.00077) \\
{[0.221]}\end{array}$ & $\begin{array}{c}-0.00047 \\
(0.00078) \\
{[-0.600]}\end{array}$ & $\begin{array}{c}-0.00029 \\
(0.00082) \\
{[-0.352]}\end{array}$ & $\begin{array}{c}-0.00106 \\
(0.00074) \\
{[-1.436]}\end{array}$ & $\begin{array}{c}-0.00139 \\
(0.00080) \\
{[-1.72]^{* * *}}\end{array}$ & $\begin{array}{c}0.00057 \\
(0.00077) \\
{[0.746]}\end{array}$ & $\begin{array}{c}-0.00082 \\
(0.00078) \\
{[-1.054]}\end{array}$ & $\begin{array}{c}-0.00059 \\
(0.00080) \\
{[-0.732]}\end{array}$ & $\begin{array}{c}0.00178 \\
(0.00080) \\
{[2.231]^{* *}}\end{array}$ & $\begin{array}{c}1.36 \mathrm{e}-05 \\
(2.73 \mathrm{e}-06) \\
{[4.993]^{*}}\end{array}$ & $\begin{array}{c}0.1197 \\
(0.0221) \\
{[5.413]^{*}}\end{array}$ & $\begin{array}{c}0.0760 \\
(0.0280) \\
{[2.712]^{*}}\end{array}$ & $\begin{array}{c}0.7893 \\
(0.0244) \\
{[32.300]^{*}}\end{array}$ \\
\hline $\begin{array}{l}\text { PORTUGAL } \\
\text { GJR-GARCH }\end{array}$ & $\begin{array}{c}0.00103 \\
(0.00052) \\
{[1.977]^{* *}}\end{array}$ & $\begin{array}{c}0.00197 \\
(0.00058) \\
{[3.389]^{*}}\end{array}$ & $\begin{array}{c}0.00036 \\
(0.00060) \\
{[0.596]}\end{array}$ & $\begin{array}{c}0.00081 \\
(0.00046) \\
{[1.761]^{* * *}}\end{array}$ & $\begin{array}{c}0.00130 \\
(0.00046) \\
{[2.802]^{*}}\end{array}$ & $\begin{array}{c}-0.00015 \\
(0.00069) \\
{[-0.211]}\end{array}$ & $\begin{array}{c}0.00039 \\
(0.00055) \\
{[0.711]}\end{array}$ & $\begin{array}{c}0.00098 \\
(0.00049) \\
{[1.995]^{* *}}\end{array}$ & $\begin{array}{c}0.00097 \\
(0.00058) \\
{[1.676]^{* * *}}\end{array}$ & $\begin{array}{c}0.00006 \\
(0.00052) \\
{[0.106]}\end{array}$ & $\begin{array}{c}0.00072 \\
(0.00053) \\
{[1.364]}\end{array}$ & $\begin{array}{c}0.00160 \\
(0.00050) \\
{[3.226]^{*}}\end{array}$ & $\begin{array}{c}1.83 \mathrm{e}-06 \\
(5.00 \mathrm{e}-07) \\
{[3.669]^{*}}\end{array}$ & & $\begin{array}{c}0.2763 \\
(0.0581) \\
{[4.751]^{*}}\end{array}$ & $\begin{array}{c}0.8330 \\
(0.0255) \\
{[32.634]^{*}}\end{array}$ \\
\hline $\begin{array}{c}\text { RUSSIA } \\
\text { GJR-GARCH }\end{array}$ & $\begin{array}{c}0.00111 \\
(0.00096) \\
{[1.158]}\end{array}$ & $\begin{array}{c}0.00334 \\
(0.00122) \\
{[2.746]^{*}}\end{array}$ & $\begin{array}{c}0.00040 \\
(0.00124) \\
{[0.319]}\end{array}$ & $\begin{array}{c}0.00175 \\
(0.00106) \\
{[1.647]^{* * *}}\end{array}$ & $\begin{array}{c}0.00155 \\
(0.00134) \\
{[1.159]}\end{array}$ & $\begin{array}{c}0.00209 \\
(0.00122) \\
{[1.710]^{* * *}}\end{array}$ & $\begin{array}{c}0.00088 \\
(0.00119) \\
{[0.736]}\end{array}$ & $\begin{array}{c}0.00290 \\
(0.00126) \\
{[2.300]^{* *}}\end{array}$ & $\begin{array}{c}0.00002 \\
(0.00117) \\
{[0.017]}\end{array}$ & $\begin{array}{c}0.00245 \\
(0.00107) \\
{[2.290]^{* *}}\end{array}$ & $\begin{array}{c}0.00158 \\
(0.00117) \\
{[1.354]}\end{array}$ & $\begin{array}{c}0.00191 \\
(0.00098) \\
{[1.940]^{* * *}}\end{array}$ & $\begin{array}{c}2.07 \mathrm{e}-05 \\
(1.95 \mathrm{e}-06) \\
{[10.607]^{*}}\end{array}$ & $\begin{array}{c}0.1757 \\
(0.0150) \\
{[11.688]^{*}}\end{array}$ & $\begin{array}{c}0.0384 \\
(0.0161) \\
{[2.382]^{* *}}\end{array}$ & $\begin{array}{c}0.7892 \\
(0.0103) \\
{[76.414]^{*}}\end{array}$ \\
\hline $\begin{array}{l}\text { SINGAPORE } \\
\text { GJR-GARCH }\end{array}$ & $\begin{array}{c}0.00189 \\
(0.00078) \\
{[2.427]^{* *}}\end{array}$ & $\begin{array}{c}-0.00068 \\
(0.00084) \\
{[-0.813]}\end{array}$ & $\begin{array}{c}-0.00029 \\
(0.00078) \\
{[-0.372]}\end{array}$ & $\begin{array}{c}-0.00021 \\
(0.00076) \\
{[-0.277]} \\
\end{array}$ & $\begin{array}{c}-0.00022 \\
(0.00078) \\
{[-0.284]}\end{array}$ & $\begin{array}{c}-0.00059 \\
(0.00083) \\
{[-0.708]}\end{array}$ & $\begin{array}{c}-0.00095 \\
(0.00073) \\
{[-1.308]} \\
\end{array}$ & $\begin{array}{c}-0.00142 \\
(0.00081) \\
{[-1.75]^{* * *}}\end{array}$ & $\begin{array}{c}0.00046 \\
(0.00076) \\
{[0.607]} \\
\end{array}$ & $\begin{array}{c}-0.00063 \\
(0.00079) \\
{[-0.802]} \\
\end{array}$ & $\begin{array}{c}-0.00084 \\
(0.00079) \\
{[-1.060]}\end{array}$ & $\begin{array}{c}0.00187 \\
(0.00080) \\
{[2.349]^{* *}} \\
\end{array}$ & $\begin{array}{c}1.34 \mathrm{e}-05 \\
(2.76 \mathrm{e}-06) \\
{[5.001]^{*}}\end{array}$ & $\begin{array}{c}0.1213 \\
(0.0222) \\
{[5.451]^{*}} \\
\end{array}$ & $\begin{array}{c}0.0731 \\
(0.0280) \\
{[2.613]^{*}}\end{array}$ & $\begin{array}{c}0.7900 \\
(0.0243) \\
{[32.551]^{*}}\end{array}$ \\
\hline $\begin{array}{l}\text { SOUTH KOREA } \\
\text { GJR-GARCH }\end{array}$ & $\begin{array}{c}0.00020 \\
(0.00093) \\
{[0.214]} \\
\end{array}$ & $\begin{array}{c}0.00143 \\
(0.00100) \\
{[1.434]}\end{array}$ & $\begin{array}{c}-0.00020 \\
(0.00099) \\
{[-0.198]}\end{array}$ & $\begin{array}{c}0.00165 \\
(0.00090) \\
{[1.838]^{* * *}}\end{array}$ & $\begin{array}{c}0.00164 \\
(0.00098) \\
{[1.677]^{* * *}}\end{array}$ & $\begin{array}{c}0.00019 \\
(0.00094) \\
{[0.198]} \\
\end{array}$ & $\begin{array}{c}0.00111 \\
(0.00090) \\
{[1.232]} \\
\end{array}$ & $\begin{array}{c}0.00044 \\
(0.00089) \\
{[0.501]} \\
\end{array}$ & $\begin{array}{c}0.00040 \\
(0.00090) \\
{[0.440]}\end{array}$ & $\begin{array}{c}0.00037 \\
(0.00108) \\
{[0.341]} \\
\end{array}$ & $\begin{array}{c}0.00274 \\
(0.00102) \\
{[2.681]^{*}} \\
\end{array}$ & $\begin{array}{c}0.00062 \\
(0.00101) \\
{[0.613]} \\
\end{array}$ & $\begin{array}{c}2.27 \mathrm{e}-06 \\
(7.40 \mathrm{e}-07) \\
{[3.067]^{*}}\end{array}$ & $\begin{array}{c}0.0400 \\
(0.0109) \\
{[3.669]^{*}} \\
\end{array}$ & $\begin{array}{c}0.0606 \\
(0.0139) \\
{[4.348]^{*}}\end{array}$ & $\begin{array}{c}0.9256 \\
(0.0095) \\
{[97.619]^{*}}\end{array}$ \\
\hline $\begin{array}{c}\text { SPAIN } \\
\text { EGARCH }\end{array}$ & $\begin{array}{c}0.00051 \\
(0.00052) \\
{[0.975]}\end{array}$ & $\begin{array}{c}0.00169 \\
(0.00051) \\
{[3.303]^{*}}\end{array}$ & $\begin{array}{c}-0.00030 \\
(0.00061) \\
{[-0.487]}\end{array}$ & $\begin{array}{c}0.00083 \\
(0.00060) \\
{[1.371]}\end{array}$ & $\begin{array}{c}0.00045 \\
(0.00056) \\
{[0.801]}\end{array}$ & $\begin{array}{c}0.00016 \\
(0.00055) \\
{[0.293]}\end{array}$ & $\begin{array}{c}-0.00014 \\
(0.00054) \\
{[-0.254]}\end{array}$ & $\begin{array}{c}-0.00054 \\
(0.000570 \\
{[-0.947]}\end{array}$ & $\begin{array}{c}0.00127 \\
(0.00048) \\
{[2.673]^{*}}\end{array}$ & $\begin{array}{c}0.00088 \\
(0.00055) \\
{[1.604]}\end{array}$ & $\begin{array}{c}0.00118 \\
(0.00053) \\
{[2.226]^{* *}}\end{array}$ & $\begin{array}{c}0.00135 \\
(0.00058) \\
{[2.350]^{* *}}\end{array}$ & $\begin{array}{c}-0.2737 \\
(0.0392) \\
{[-6.989]^{*}}\end{array}$ & $\begin{array}{c}0.1694 \\
(0.0178) \\
{[9.518]^{*}}\end{array}$ & $\begin{array}{c}-0.0757 \\
(0.0101) \\
{[-7.477]^{*}}\end{array}$ & $\begin{array}{c}0.9842 \\
(0.0036) \\
{[271.77]^{*}}\end{array}$ \\
\hline $\begin{array}{c}\text { SRI LANKA } \\
\text { EGARCH }\end{array}$ & $\begin{array}{c}-0.00002 \\
(0.00051) \\
{[-0.038]}\end{array}$ & $\begin{array}{c}0.00125 \\
(0.00049) \\
{[2.575]^{*}}\end{array}$ & $\begin{array}{c}-0.00041 \\
(0.00042) \\
{[-0.961]}\end{array}$ & $\begin{array}{c}0.00061 \\
(0.00052) \\
{[1.171]}\end{array}$ & $\begin{array}{c}0.00036 \\
(0.00048) \\
{[0.751]}\end{array}$ & $\begin{array}{c}-0.00023 \\
(0.00048) \\
{[-0.487]}\end{array}$ & $\begin{array}{c}0.00052 \\
(0.00043) \\
{[1.203]}\end{array}$ & $\begin{array}{c}-0.00082 \\
(0.00046) \\
{[-1.80]^{* * *}}\end{array}$ & $\begin{array}{c}0.00143 \\
(0.00044) \\
{[3.292]^{*}}\end{array}$ & $\begin{array}{c}-0.00011 \\
(0.00047) \\
{[-0.240]}\end{array}$ & $\begin{array}{c}0.00054 \\
(0.00053) \\
{[1.022]}\end{array}$ & $\begin{array}{c}0.00060 \\
(0.00051) \\
{[1.190]}\end{array}$ & $\begin{array}{c}-1.6124 \\
(0.1751) \\
{[-9.210]^{*}}\end{array}$ & $\begin{array}{c}0.5905 \\
(0.0435) \\
{[13.566]^{*}}\end{array}$ & $\begin{array}{l}-0.0358 \\
(0.0238) \\
{[-1.501]}\end{array}$ & $\begin{array}{c}0.8729 \\
(0.0173) \\
{[50.323]^{*}}\end{array}$ \\
\hline
\end{tabular}


Table 3. (cont.) GARCH estimations of equation (2)

\begin{tabular}{|c|c|c|c|c|c|c|c|c|c|c|c|c|c|c|c|c|}
\hline Countries & $\beta_{1}$ & $\beta_{2}$ & $\beta_{3}$ & $\beta_{4}$ & $\beta_{5}$ & $\beta_{6}$ & $\boldsymbol{\beta}_{7}$ & $\beta_{8}$ & $\beta_{9}$ & $\beta_{10}$ & $\beta_{11}$ & $\beta_{12}$ & $\omega$ & $\boldsymbol{\alpha}_{0}$ & $\gamma$ & $\boldsymbol{\alpha}_{1}$ \\
\hline $\begin{array}{l}\text { SWEDEN } \\
\text { GJR-GARCH }\end{array}$ & $\begin{array}{c}0.00047 \\
(0.00063) \\
{[0.754]}\end{array}$ & $\begin{array}{c}0.00152 \\
(0.00064) \\
{[2.390]^{* *}}\end{array}$ & $\begin{array}{c}0.00004 \\
(0.00080) \\
{[0.054]}\end{array}$ & $\begin{array}{c}0.00017 \\
(0.00067) \\
{[0.246]}\end{array}$ & $\begin{array}{c}-0.00028 \\
(0.00069) \\
{[-0.405]}\end{array}$ & $\begin{array}{c}-0.00077 \\
(0.00082) \\
{[-0.948]}\end{array}$ & $\begin{array}{c}0.00042 \\
(0.00069) \\
{[0.604]}\end{array}$ & $\begin{array}{c}-0.00040 \\
(0.00067) \\
{[-0.603]}\end{array}$ & $\begin{array}{c}0.00114 \\
(0.00066) \\
{[1.722]^{* * *}}\end{array}$ & $\begin{array}{c}0.00003 \\
(0.00070) \\
{[0.041]}\end{array}$ & $\begin{array}{c}0.00061 \\
(0.00079) \\
{[0.767]}\end{array}$ & $\begin{array}{c}0.00030 \\
(0.00080) \\
{[0.375]}\end{array}$ & $\begin{array}{c}1.71 \mathrm{e}-06 \\
(2.89 \mathrm{e}-07) \\
{[5.913]^{*}}\end{array}$ & $\begin{array}{c}-0.0163 \\
(0.0082) \\
{[-1.993]^{* *}}\end{array}$ & $\begin{array}{c}0.1265 \\
(0.0121) \\
{[10.484]^{*}}\end{array}$ & $\begin{array}{c}0.9402 \\
(0.0075) \\
{[124.87]^{*}}\end{array}$ \\
\hline $\begin{array}{c}\text { SWITZERLAND } \\
\text { EGARCH }\end{array}$ & $\begin{array}{c}0.00036 \\
(0.00041) \\
{[0.889]}\end{array}$ & $\begin{array}{c}0.00108 \\
(0.00040) \\
{[2.689]^{*}}\end{array}$ & $\begin{array}{c}0.00033 \\
(0.00044) \\
{[0.755]}\end{array}$ & $\begin{array}{c}0.00023 \\
(0.00044) \\
{[0.536]}\end{array}$ & $\begin{array}{c}0.00084 \\
(0.00042) \\
{[1.994]^{* *}}\end{array}$ & $\begin{array}{c}-0.00004 \\
(0.00039) \\
{[-0.106]}\end{array}$ & $\begin{array}{c}0.00078 \\
(0.00039) \\
{[2.026]^{* *}}\end{array}$ & $\begin{array}{c}0.00013 \\
(0.00041) \\
{[0.324]}\end{array}$ & $\begin{array}{c}0.00030 \\
(0.00041) \\
{[0.724]}\end{array}$ & $\begin{array}{c}0.00070 \\
(0.00045) \\
{[1.544]}\end{array}$ & $\begin{array}{c}0.00069 \\
(0.00043) \\
{[1.608]}\end{array}$ & $\begin{array}{c}0.00142 \\
(0.00041) \\
{[3.443]^{*}}\end{array}$ & $\begin{array}{c}-0.3229 \\
(0.0414) \\
{[-7.805]^{*}}\end{array}$ & $\begin{array}{c}0.1516 \\
(0.0162) \\
{[9.334]^{*}}\end{array}$ & $\begin{array}{c}-0.0868 \\
(0.0094) \\
{[-9.222]^{*}}\end{array}$ & $\begin{array}{c}0.9781 \\
(0.0037) \\
{[261.73]^{*}}\end{array}$ \\
\hline $\begin{array}{l}\text { TAIWAN } \\
\text { EGARCH }\end{array}$ & $\begin{array}{c}0.00104 \\
(0.00080) \\
{[1.307]}\end{array}$ & $\begin{array}{c}0.00136 \\
(0.00091) \\
{[1.496]}\end{array}$ & $\begin{array}{c}-0.00015 \\
(0.00081) \\
{[-0.186]}\end{array}$ & $\begin{array}{c}-0.00009 \\
(0.00079) \\
{[-0.108]}\end{array}$ & $\begin{array}{c}-0.00019 \\
(0.00078) \\
{[-0.238]}\end{array}$ & $\begin{array}{c}0.00149 \\
(0.00074) \\
{[2.009]^{* *}}\end{array}$ & $\begin{array}{c}0.00006 \\
(0.00072) \\
{[0.080]}\end{array}$ & $\begin{array}{c}-0.00043 \\
(0.00080) \\
{[-0.542]}\end{array}$ & $\begin{array}{c}-0.00118 \\
(0.00080) \\
{[-1.477]}\end{array}$ & $\begin{array}{c}0.00006 \\
(0.00078) \\
{[0.082]}\end{array}$ & $\begin{array}{c}0.00110 \\
(0.00080) \\
{[1.382]}\end{array}$ & $\begin{array}{c}0.00112 \\
(0.00081) \\
{[1.389]}\end{array}$ & $\begin{array}{c}-0.2839 \\
(0.0451) \\
{[-6.297]^{*}}\end{array}$ & & $\begin{array}{c}-0.0753 \\
(0.0112) \\
{[-6.743]^{*}}\end{array}$ & $\begin{array}{c}0.9796 \\
(0.0045) \\
{[216.25]^{*}}\end{array}$ \\
\hline $\begin{array}{l}\text { THAILAND } \\
\text { GJR-GARCH }\end{array}$ & $\begin{array}{c}0.00265 \\
(0.00129) \\
{[2.058]^{* *}} \\
\end{array}$ & $\begin{array}{c}-0.00151 \\
(0.00137) \\
{[-1.097]}\end{array}$ & $\begin{array}{c}-0.00276 \\
(0.00137) \\
{[-2.019]^{* *}}\end{array}$ & $\begin{array}{c}0.00004 \\
(0.00135) \\
{[0.027]}\end{array}$ & $\begin{array}{c}0.00055 \\
(0.00146) \\
{[0.377]} \\
\end{array}$ & $\begin{array}{c}0.00129 \\
(0.00142) \\
{[0.912]} \\
\end{array}$ & $\begin{array}{c}-0.00340 \\
(0.00143) \\
{[-2.372]^{* *}}\end{array}$ & $\begin{array}{c}-0.00092 \\
(0.00132) \\
{[-0.696]}\end{array}$ & $\begin{array}{c}-0.00156 \\
(0.00130) \\
{[-1.202]}\end{array}$ & $\begin{array}{c}0.00079 \\
(0.00136) \\
{[0.577]} \\
\end{array}$ & $\begin{array}{c}0.00065 \\
(0.00129) \\
{[0.506]}\end{array}$ & $\begin{array}{c}0.00131 \\
(0.00146) \\
{[0.896]} \\
\end{array}$ & $\begin{array}{c}1.53 \mathrm{e}-05 \\
(4.48 \mathrm{e}-06) \\
{[3.426]^{*}}\end{array}$ & & $\begin{array}{c}0.0744 \\
(0.0304) \\
{[2.445]^{* *}} \\
\end{array}$ & $\begin{array}{c}0.8381 \\
(0.0270) \\
{[31.087]^{*}}\end{array}$ \\
\hline $\begin{array}{l}\text { TURKEY } \\
\text { GARCH }\end{array}$ & $\begin{array}{c}0.0074 \\
(0.00147) \\
{[0.545]} \\
\end{array}$ & $\begin{array}{c}0.00112 \\
(0.00148) \\
{[0.757]} \\
\end{array}$ & $\begin{array}{c}-0.00148 \\
(0.00141) \\
{[-1.047]}\end{array}$ & $\begin{array}{c}0.00282 \\
(0.00168) \\
{[1.680]^{* * *}}\end{array}$ & $\begin{array}{c}-0.00128 \\
(0.00151) \\
{[-0.845]}\end{array}$ & $\begin{array}{c}-0.00028 \\
(0.00147) \\
{[-0.195]}\end{array}$ & $\begin{array}{c}0.0024 \\
(0.0013) \\
{[1.851]^{* * *}}\end{array}$ & $\begin{array}{c}-1.13 \mathrm{e}-05 \\
(0.0015) \\
{[-0.007]} \\
\end{array}$ & $\begin{array}{c}0.00342 \\
(0.0011) \\
{[3.116]^{*}} \\
\end{array}$ & $\begin{array}{c}0.00351 \\
(0.00105) \\
{[3.323]^{*}} \\
\end{array}$ & $\begin{array}{c}0.00058 \\
(0.00148) \\
{[0.395]} \\
\end{array}$ & $\begin{array}{c}0.003 \\
(0.00176) \\
{[1.698]^{* * *}}\end{array}$ & $\begin{array}{c}8.65 \mathrm{e}-06 \\
(1.90 \mathrm{e}-06) \\
{[4.540]^{*}}\end{array}$ & $\begin{array}{c}0.1029 \\
(0.0083) \\
{[12.305]^{*}}\end{array}$ & & $\begin{array}{c}0.8920 \\
(0.0076) \\
{[116.93]^{*}}\end{array}$ \\
\hline $\begin{array}{l}\text { UK-FTSE } 100 \\
\text { GJR-GARCH }\end{array}$ & $\begin{array}{c}0.00054 \\
(0.00033) \\
{[1.670]^{* * *}}\end{array}$ & $\begin{array}{c}0.00047 \\
(0.00036) \\
{[1.319]}\end{array}$ & $\begin{array}{c}0.00023 \\
(0.00034) \\
{[0.675]}\end{array}$ & $\begin{array}{c}0.00047 \\
(0.00038) \\
{[1.225]}\end{array}$ & $\begin{array}{c}0.00016 \\
(0.00036) \\
{[0.456]}\end{array}$ & $\begin{array}{c}-0.00008 \\
(0.00034) \\
{[-0.249]}\end{array}$ & $\begin{array}{c}0.00042 \\
(0.00035) \\
{[1.208]}\end{array}$ & $\begin{array}{c}0.00068 \\
(0.00037) \\
{[1.843]^{* * *}}\end{array}$ & $\begin{array}{c}-0.00009 \\
(0.00035) \\
{[-0.263]}\end{array}$ & $\begin{array}{c}0.00050 \\
(0.00036) \\
{[1.390]}\end{array}$ & $\begin{array}{c}0.00035 \\
(0.00037) \\
{[0.950]}\end{array}$ & $\begin{array}{c}0.00101 \\
(0.00038) \\
{[2.637]^{*}}\end{array}$ & $\begin{array}{c}1.62 \mathrm{e}-06 \\
(2.64 \mathrm{e}-07) \\
{[6.120]^{*}}\end{array}$ & & $\begin{array}{c}0.0786 \\
(0.0104) \\
{[7.560]^{*}}\end{array}$ & $\begin{array}{c}0.9087 \\
(0.0078) \\
{[116.82]^{*}}\end{array}$ \\
\hline $\begin{array}{c}\text { UK-FTSE } 250 \\
\text { EGARCH }\end{array}$ & $\begin{array}{c}0.00027 \\
(0.00037) \\
{[0.734]} \\
\end{array}$ & $\begin{array}{c}0.00075 \\
(0.00047) \\
{[1.589]} \\
\end{array}$ & $\begin{array}{c}0.00025 \\
(0.00052) \\
{[0.471]} \\
\end{array}$ & $\begin{array}{c}0.00036 \\
(0.00050) \\
{[0.717]}\end{array}$ & $\begin{array}{c}0.00088 \\
(0.00048) \\
{[1.822]^{* * *}}\end{array}$ & $\begin{array}{c}0.00026 \\
(0.00052) \\
{[0.497]} \\
\end{array}$ & $\begin{array}{c}0.00009 \\
(0.00047) \\
{[0.180]} \\
\end{array}$ & $\begin{array}{c}0.00123 \\
(0.00051) \\
{[2.435]^{*}}\end{array}$ & $\begin{array}{c}0.00007 \\
(0.00046) \\
{[0.146]} \\
\end{array}$ & $\begin{array}{c}0.00067 \\
(0.00058) \\
{[1.160]} \\
\end{array}$ & $\begin{array}{c}0.00073 \\
(0.00054) \\
{[1.353]} \\
\end{array}$ & $\begin{array}{c}0.00126 \\
(0.00055) \\
{[2.281]^{*}}\end{array}$ & & & $\begin{array}{c}-0.0863 \\
(0.0134) \\
{[-6.451]^{*}}\end{array}$ & $\begin{array}{c}0.9749 \\
(0.0054) \\
{[181.42]^{*}}\end{array}$ \\
\hline $\begin{array}{c}\text { US DOW } \\
\text { JONES } \\
\text { COMPOSITE } \\
\text { EGARCH }\end{array}$ & $\begin{array}{l}0.00085 \\
0.00028 \\
(3.002)^{*}\end{array}$ & $\begin{array}{c}0.00056 \\
(0.00031) \\
{[1.794]^{* * *}}\end{array}$ & $\begin{array}{c}0.00031 \\
(0.00029) \\
{[1.052]}\end{array}$ & $\begin{array}{c}0.00060 \\
(0.00032) \\
{[1.893]^{* * *}}\end{array}$ & $\begin{array}{c}0.00042 \\
(0.00030) \\
{[1.388]}\end{array}$ & $\begin{array}{c}-0.00007 \\
(0.00030) \\
{[-0.247]}\end{array}$ & $\begin{array}{c}0.00055 \\
(0.00030) \\
{[1.833]^{* * *}}\end{array}$ & $\begin{array}{c}0.00015 \\
(0.00030) \\
{[0.509]}\end{array}$ & $\begin{array}{c}-0.00036 \\
(0.00031) \\
{[-1.167]}\end{array}$ & $\begin{array}{c}0.00083 \\
(0.00030) \\
{[2.801]^{*}}\end{array}$ & $\begin{array}{c}0.00073 \\
(0.00032) \\
{[2.251]^{* *}}\end{array}$ & $\begin{array}{c}0.00049 \\
(0.00030) \\
{[1.631]}\end{array}$ & $\begin{array}{l}-0.2232 \\
(0.0278) \\
{[-8.018]^{*}}\end{array}$ & & $\begin{array}{c}-0.0579 \\
(0.0068) \\
{[-8.493]^{*}}\end{array}$ & $\begin{array}{c}0.9846 \\
(0.0026) \\
{[384.87]^{*}}\end{array}$ \\
\hline $\begin{array}{c}\text { US NASDAQ } \\
100 \\
\text { EGARCH }\end{array}$ & $\begin{array}{c}0.00148 \\
(0.00026) \\
{[5.726]^{*}}\end{array}$ & $\begin{array}{c}0.00080 \\
(0.00027) \\
{[2.935]^{*}}\end{array}$ & $\begin{array}{c}0.00087 \\
(0.00024) \\
{[3.568]^{*}}\end{array}$ & $\begin{array}{c}0.00117 \\
(0.00023) \\
{[5.023]^{*}}\end{array}$ & $\begin{array}{c}0.00081 \\
(0.00024) \\
{[3.333]^{*}}\end{array}$ & $\begin{array}{c}0.00062 \\
(0.00024) \\
{[2.616]^{*}}\end{array}$ & $\begin{array}{c}0.00056 \\
(0.00023) \\
{[2.469]^{* *}}\end{array}$ & $\begin{array}{c}0.00072 \\
(0.00024) \\
{[3.052]^{*}}\end{array}$ & $\begin{array}{c}0.00030 \\
(0.00024) \\
{[1.263]}\end{array}$ & $\begin{array}{c}0.00046 \\
(0.00025) \\
{[1.849]^{* * *}}\end{array}$ & $\begin{array}{c}0.00125 \\
(0.00028) \\
{[4.508]^{*}}\end{array}$ & $\begin{array}{c}0.00104 \\
(0.00026) \\
{[3.983]^{*}}\end{array}$ & & & & $\begin{array}{c}0.9905 \\
(0.0015) \\
{[645.22]^{*}}\end{array}$ \\
\hline $\begin{array}{c}\text { US NEW YORK } \\
\text { COMPOSITE } \\
\text { EGARCH }\end{array}$ & $\begin{array}{c}0.00062 \\
(0.00020) \\
{[3.106]^{*}}\end{array}$ & $\begin{array}{c}0.00028 \\
(0.00022) \\
{[1.292]}\end{array}$ & $\begin{array}{c}0.00045 \\
(0.00021) \\
{[2.132]^{* *}}\end{array}$ & $\begin{array}{c}0.00062 \\
(0.00022) \\
{[2.816]^{*}}\end{array}$ & $\begin{array}{c}0.00026 \\
(0.00022) \\
{[1.199]}\end{array}$ & $\begin{array}{c}-0.00004 \\
(0.00022) \\
{[-0.173]}\end{array}$ & $\begin{array}{c}0.00027 \\
(0.00022) \\
{[1.221]}\end{array}$ & $\begin{array}{c}0.00022 \\
(0.00021) \\
{[1.035]}\end{array}$ & $\begin{array}{c}0.00021 \\
(0.00021) \\
{[0.997]}\end{array}$ & $\begin{array}{c}0.00025 \\
(0.00021) \\
{[1.195]}\end{array}$ & $\begin{array}{c}0.00076 \\
(0.00024) \\
{[3.209]^{*}}\end{array}$ & $\begin{array}{c}0.00060 \\
(0.00022) \\
{[2.706]^{*}}\end{array}$ & $\begin{array}{c}-0.2259 \\
(0.0199) \\
{[-11.338]^{*}}\end{array}$ & $\begin{array}{c}0.1144 \\
(0.0083) \\
{[13.760]^{*}}\end{array}$ & $\begin{array}{c}-0.0653 \\
(0.0050) \\
{[-12.971]^{*}}\end{array}$ & $\begin{array}{c}0.9857 \\
(0.0018) \\
{[562.61]^{*}}\end{array}$ \\
\hline $\begin{array}{l}\text { US - S\&P } 500 \\
\text { EGARCH }\end{array}$ & $\begin{array}{c}0.00061 \\
(0.00017) \\
{[3.508]^{*}}\end{array}$ & $\begin{array}{c}-0.00003 \\
(0.00018) \\
{[-0.162]}\end{array}$ & $\begin{array}{c}0.00041 \\
(0.00017) \\
{[2.503]^{* *}}\end{array}$ & $\begin{array}{c}0.00075 \\
(0.00017) \\
{[4.404]^{*}}\end{array}$ & $\begin{array}{c}0.00037 \\
(0.00017) \\
{[2.175]^{* *}}\end{array}$ & $\begin{array}{c}0.00007 \\
(0.00018) \\
{[0.390]}\end{array}$ & $\begin{array}{c}0.00056 \\
(0.00018) \\
{[3.092]^{*}}\end{array}$ & $\begin{array}{c}0.00014 \\
(0.00018) \\
{[0.783]}\end{array}$ & $\begin{array}{c}0.00034 \\
(0.00017) \\
{[2.018]^{* *}}\end{array}$ & $\begin{array}{c}0.00041 \\
(0.00017) \\
{[2.347]^{* *}}\end{array}$ & $\begin{array}{c}0.00072 \\
(0.00019) \\
{[3.695]^{*}}\end{array}$ & $\begin{array}{c}0.00053 \\
(0.00018) \\
{[2.946]^{*}}\end{array}$ & $\begin{array}{c}-0.2107 \\
(0.0158) \\
{[-13.305]^{*}}\end{array}$ & $\begin{array}{c}0.1235 \\
(0.0072) \\
{[17.166]^{*}}\end{array}$ & $\begin{array}{c}-0.0663 \\
(0.0044) \\
{[-15.086]^{*}}\end{array}$ & $\begin{array}{c}0.9881 \\
(0.0014) \\
{[721.77]^{*}}\end{array}$ \\
\hline $\begin{array}{l}\text { YUGOSLAVIA } \\
\text { GJR-GARCH }\end{array}$ & $\begin{array}{c}0.00142 \\
(0.00121) \\
{[1.169]}\end{array}$ & $\begin{array}{c}0.00126 \\
(0.00115) \\
{[1.098]}\end{array}$ & $\begin{array}{c}0.00104 \\
(0.00115) \\
{[0.910]}\end{array}$ & $\begin{array}{c}-0.00080 \\
(0.00133) \\
{[-0.600]}\end{array}$ & $\begin{array}{c}-0.00168 \\
(0.00123) \\
{[-1.375]}\end{array}$ & $\begin{array}{c}-0.00230 \\
(0.00106) \\
{[-2.165]^{* *}}\end{array}$ & $\begin{array}{c}0.00172 \\
(0.00116) \\
{[1.485]}\end{array}$ & $\begin{array}{c}-0.00068 \\
(0.00095) \\
{[-0.711]}\end{array}$ & $\begin{array}{c}-0.00106 \\
(0.00122) \\
{[-0.868]}\end{array}$ & $\begin{array}{c}-0.00004 \\
(0.00084) \\
{[-0.048]}\end{array}$ & $\begin{array}{c}0.00066 \\
(0.00102) \\
{[0.643]}\end{array}$ & $\begin{array}{c}0.00196 \\
(0.00097) \\
{[2.022]^{* *}}\end{array}$ & $\begin{array}{c}1.54 \mathrm{e}-05 \\
(4.39 \mathrm{e}-06) \\
{[3.498]^{*}}\end{array}$ & $\begin{array}{c}0.4828 \\
(0.1048) \\
{[4.608]^{*}}\end{array}$ & $\begin{array}{c}0.0433 \\
(0.1182) \\
{[0.366]}\end{array}$ & $\begin{array}{c}0.5110 \\
(0.0582) \\
{[8.775]^{*}}\end{array}$ \\
\hline $\begin{array}{l}\text { ZAMBIA } \\
\text { EGARCH }\end{array}$ & $\begin{array}{c}0.00278 \\
(0.00034) \\
{[8.284]^{*}}\end{array}$ & $\begin{array}{c}0.00375 \\
(0.00042) \\
{[8.910]^{*}}\end{array}$ & $\begin{array}{c}0.00053 \\
(0.00072) \\
{[0.728]}\end{array}$ & $\begin{array}{c}0.00109 \\
(0.00027) \\
{[4.020]^{*}}\end{array}$ & $\begin{array}{c}0.00164 \\
(0.00033) \\
{[4.973]^{*}}\end{array}$ & $\begin{array}{c}0.00145 \\
(0.00050) \\
{[2.907]^{*}} \\
\end{array}$ & $\begin{array}{c}-0.00039 \\
(0.00052) \\
{[-0.752]}\end{array}$ & $\begin{array}{c}0.00388 \\
(0.00039) \\
{[9.914]^{*}}\end{array}$ & $\begin{array}{c}0.00008 \\
(0.00107) \\
{[0.077]}\end{array}$ & $\begin{array}{c}-0.00108 \\
(0.00049) \\
{[-2.200]^{* *}}\end{array}$ & $\begin{array}{c}0.00096 \\
(0.00056) \\
{[1.703]^{* * *}}\end{array}$ & $\begin{array}{c}0.00001 \\
(0.00063) \\
{[0.009]}\end{array}$ & $\begin{array}{c}-0.9933 \\
(0.0805) \\
{[-12.334]^{*}}\end{array}$ & $\begin{array}{c}0.2945 \\
(0.0179) \\
{[16.420]^{*}}\end{array}$ & $\begin{array}{c}-0.0818 \\
(0.0150) \\
{[-5.454]^{*}}\end{array}$ & $\begin{array}{c}0.9075 \\
(0.0084) \\
{[107.67]^{*}}\end{array}$ \\
\hline
\end{tabular}

*denotes significance in 0.01 level, **denotes significance in 0.05 level *** denotes significance in 0.10 level - standard errors in parentheses, $\mathrm{z}$-statistics in brackets 
Table 4. Diagnostic tests of GARCH estimations

\begin{tabular}{|c|c|c|c|c|c|c|c|c|c|c|c|c|c|}
\hline Countries & $\mathrm{R}^{2}$ adj. & AIC & SBC & $\mathbf{L L}$ & $\mathrm{LBQ}^{2}(12)$ & $\begin{array}{l}\text { ARCH- } \\
\text { LM (5) }\end{array}$ & Countries & $\mathrm{R}^{2}$ adj. & AIC & SBC & $\mathbf{L L}$ & $\mathrm{LBQ}^{2}(12)$ & $\begin{array}{l}\text { ARCH- } \\
\text { LM (5) }\end{array}$ \\
\hline $\begin{array}{l}\text { ARGENTINA } \\
\text { GJR-GARCH }\end{array}$ & 0.0001905 & -5.160 & -5.125 & 7664.433 & $\begin{array}{l}14.161 \\
\{0.291\}\end{array}$ & $\begin{array}{c}1.102 \\
\{0.3562\}\end{array}$ & $\begin{array}{c}\text { INDIA } \\
\text { GJR-GARCH }\end{array}$ & 0.001094 & -5.593 & -5.557 & 7808.594 & $\begin{array}{c}9.204 \\
\{0.685\}\end{array}$ & $\begin{array}{c}0.332 \\
\{0.8935\}\end{array}$ \\
\hline $\begin{array}{l}\text { AUSTRALIA } \\
\text { EGARCH }\end{array}$ & -0.000303 & -6.890 & -6.871 & 21081.61 & $\begin{array}{l}6.9416 \\
\{0.861\}\end{array}$ & $\begin{array}{c}1.389 \\
\{0.2246\}\end{array}$ & $\begin{array}{l}\text { INDONESIA } \\
\text { GJR-GARCH }\end{array}$ & 0.005046 & -5.555 & -5.518 & 7608.255 & $\begin{array}{c}5.217 \\
\{0.950\}\end{array}$ & $\begin{array}{c}0.418 \\
\{0.8365\}\end{array}$ \\
\hline $\begin{array}{c}\text { AUSTRIA } \\
\text { GJR-GARCH }\end{array}$ & 0.004281 & -6.418 & -6.391 & 12622.61 & $\begin{array}{c}9.651 \\
\{0.646\}\end{array}$ & $\begin{array}{c}0.780 \\
\{0.5634\}\end{array}$ & $\begin{array}{l}\text { IRELAND } \\
\text { EGARCH }\end{array}$ & -0.000083 & -6.721 & -6.703 & 22001.48 & $\begin{array}{l}1047.8 \\
\{0.000\}\end{array}$ & $\begin{array}{l}107.472 \\
\{0.000\}\end{array}$ \\
\hline $\begin{array}{c}\text { BELGIUM } \\
\text { EGARCH }\end{array}$ & -0.004098 & -6.602 & -6.515 & 3117.079 & $\begin{array}{r}17.508 \\
\{0.131\} \\
\end{array}$ & $\begin{array}{c}1.459 \\
\{0.2007\} \\
\end{array}$ & $\begin{array}{c}\text { ISRAEL } \\
\text { EGARCH }\end{array}$ & 0.005664 & -5.796 & -5.754 & 6770.230 & $\begin{array}{l}13.675 \\
\{0.316\} \\
\end{array}$ & $\begin{array}{c}1.833 \\
\{0.1029\} \\
\end{array}$ \\
\hline $\begin{array}{c}\text { BRAZIL } \\
\text { GJR-GARCH } \\
\end{array}$ & 0.000555 & -4.878 & -4.850 & 9327.323 & $\begin{array}{l}15.103 \\
\{0.236\} \\
\end{array}$ & $\begin{array}{c}1.355 \\
\{0.2380\} \\
\end{array}$ & $\begin{array}{c}\text { ITALY } \\
\text { EGARCH }\end{array}$ & 0.000296 & -6.491 & -6.448 & 7326.350 & $\begin{array}{l}10.437 \\
\{0.578\} \\
\end{array}$ & $\begin{array}{c}0.694 \\
\{0.6278\}\end{array}$ \\
\hline $\begin{array}{l}\text { CANADA } \\
\text { EGARCH }\end{array}$ & -0.000579 & -6.542 & -6.498 & 7252.581 & $\begin{array}{l}13.640 \\
\{0.324\}\end{array}$ & $\begin{array}{c}0.423 \\
\{0.8324\}\end{array}$ & $\begin{array}{c}\text { JAPAN } \\
\text { EGARCH }\end{array}$ & -0.000533 & -6.066 & -6.047 & 18485.11 & $\begin{array}{c}5.360 \\
\{0.945\}\end{array}$ & $\begin{array}{c}0.927 \\
\{0.4616\}\end{array}$ \\
\hline $\begin{array}{c}\text { CHILE } \\
\text { EGARCH } \\
\end{array}$ & 0.001048 & -6.672 & -6.599 & 4007.030 & $\begin{array}{r}13.369 \\
\{0.343\} \\
\end{array}$ & $\begin{array}{c}0.939 \\
\{0.4544\} \\
\end{array}$ & $\begin{array}{l}\text { JORDAN } \\
\text { EGARCH } \\
\end{array}$ & -0.002495 & -6.943 & -6.917 & 14262.29 & $\begin{array}{c}6.581 \\
\{0.884\} \\
\end{array}$ & $\begin{array}{c}0.839 \\
\{0.5215\} \\
\end{array}$ \\
\hline $\begin{array}{l}\text { CHINA } \\
\text { EGARCH }\end{array}$ & -0.000383 & -5.723 & -5.685 & 7539.898 & $\begin{array}{c}8.779 \\
\{0.722\}\end{array}$ & $\begin{array}{c}0.197 \\
\{0.9635\}\end{array}$ & $\begin{array}{l}\text { KUWAIT } \\
\text { EGARCH }\end{array}$ & 0.000475 & -6.912 & -6.862 & 6552.565 & $\begin{array}{c}7.943 \\
\{0.790\}\end{array}$ & $\begin{array}{c}0.369 \\
\{0.8698\}\end{array}$ \\
\hline $\begin{array}{l}\text { CROATIA } \\
\text { EGARCH }\end{array}$ & 0.001665 & -5.856 & -5.821 & 8569.988 & $\begin{array}{c}1.315 \\
\{1.000\}\end{array}$ & $\begin{array}{c}0.125 \\
\{0.9867\}\end{array}$ & $\begin{array}{l}\text { LATVIA } \\
\text { EGARCH }\end{array}$ & 0.002417 & -6.401 & -6.357 & 7163.862 & $\begin{array}{c}3.220 \\
\{0.994\}\end{array}$ & $\begin{array}{c}0.294 \\
\{0.9160\}\end{array}$ \\
\hline $\begin{array}{l}\text { DENMARK } \\
\text { GJR-GARCH }\end{array}$ & -0.000127 & -6.403 & -6.375 & 12256.91 & $\begin{array}{l}11.445 \\
\{0.490\}\end{array}$ & $\begin{array}{c}1.071 \\
\{0.3741\}\end{array}$ & $\begin{array}{l}\text { LITHUANIA } \\
\text { GJR-GARCH }\end{array}$ & -0.000020 & -6.783 & -6.738 & 7444.488 & $\begin{array}{c}1.282 \\
\{1.000\}\end{array}$ & $\begin{array}{c}0.202 \\
\{0.9614\}\end{array}$ \\
\hline $\begin{array}{l}\text { EGYPT } \\
\text { EGARCH }\end{array}$ & 0.002470 & -6.884 & -6.840 & 7596.527 & $\begin{array}{c}9.468 \\
\{0.663\}\end{array}$ & $\begin{array}{c}1.102 \\
\{0.3569\}\end{array}$ & $\begin{array}{c}\text { LUXEMBURG } \\
\text { GARCH }\end{array}$ & -0.001806 & -5.306 & -5.279 & 11316.96 & $\begin{array}{l}1.3854 \\
\{1.000\}\end{array}$ & $\begin{array}{c}0.072 \\
\{0.9963\}\end{array}$ \\
\hline $\begin{array}{l}\text { ESTONIA } \\
\text { EGARCH }\end{array}$ & 0.003567 & -6.850 & -6.796 & 5912.008 & $\begin{array}{c}5.187 \\
\{0.951\}\end{array}$ & $\begin{array}{c}0.155 \\
\{0.9784\}\end{array}$ & $\begin{array}{l}\text { MALAYSIA } \\
\text { EGARCH }\end{array}$ & 0.001960 & -6.316 & -6.287 & 11570.24 & $\begin{array}{l}8.552 \\
\{0.741\}\end{array}$ & $\begin{array}{c}1.209 \\
\{0.3020\}\end{array}$ \\
\hline $\begin{array}{l}\text { FINLAND } \\
\text { EGARCH }\end{array}$ & 0.000329 & -5.317 & -5.280 & 7084.155 & $\begin{array}{c}4.905 \\
\{0.961\}\end{array}$ & $\begin{array}{c}0.648 \\
\{0.6630\}\end{array}$ & $\begin{array}{l}\text { MEXICO } \\
\text { EGARCH }\end{array}$ & 0.001734 & -5.686 & -5.661 & 12007.48 & $\begin{array}{l}21.384 \\
\{0.045\}\end{array}$ & $\begin{array}{c}3.064 \\
\{0.0091\}\end{array}$ \\
\hline $\begin{array}{l}\text { FRANCE } \\
\text { EGARCH }\end{array}$ & 0.002269 & -6.059 & -6.035 & 14229.10 & $\begin{array}{c}8.778 \\
\{0.722\}\end{array}$ & $\begin{array}{c}1.304 \\
\{0.2589\}\end{array}$ & $\begin{array}{l}\text { NETHERLANDS } \\
\text { GJR-GARCH }\end{array}$ & 0.001468 & -6.280 & -6.253 & 12775.54 & $\begin{array}{l}11.034 \\
\{0.526\}\end{array}$ & $\begin{array}{c}1.335 \\
\{0.2458\}\end{array}$ \\
\hline $\begin{array}{c}\text { GERMANY } \\
\text { EGARCH }\end{array}$ & 0.002016 & -6.085 & -6.060 & 13717.76 & $\begin{array}{c}1.209 \\
\{1.000\}\end{array}$ & $\begin{array}{c}0.099 \\
\{0.9922\}\end{array}$ & $\begin{array}{l}\text { NEW ZEALAND } \\
\text { EGARCH }\end{array}$ & 0.002838 & -7.313 & -7.231 & 3706.905 & $\begin{array}{l}16.020 \\
\{0.190\}\end{array}$ & $\begin{array}{c}0.397 \\
\{0.8505\}\end{array}$ \\
\hline $\begin{array}{c}\text { GREECE } \\
\text { GJR-GARCH }\end{array}$ & 0.002824 & -5.653 & -5.616 & 7575.885 & $\begin{array}{l}24.876 \\
\{0.015\}\end{array}$ & $\begin{array}{c}3.231 \\
\{0.0065\}\end{array}$ & $\begin{array}{l}\text { NORWAY } \\
\text { GJR-GARCH }\end{array}$ & 0.002330 & -6.081 & -6.032 & 5870.386 & $\begin{array}{c}9 . .838 \\
\{0.630\}\end{array}$ & $\begin{array}{c}0.163 \\
\{0.9759\}\end{array}$ \\
\hline $\begin{array}{c}\text { HONG KONG } \\
\text { EGARCH }\end{array}$ & 0.000714 & -5.806 & -5.786 & 15669.69 & $\begin{array}{l}252.26 \\
\{0.000\}\end{array}$ & $\begin{array}{l}54.707 \\
\{0.000\}\end{array}$ & $\begin{array}{c}\text { PAKISTAN } \\
\text { GJR-GARCH } \\
\end{array}$ & 0.001115 & -5.676 & -5.637 & 7265.310 & $\begin{array}{c}7.209 \\
\{0.843\} \\
\end{array}$ & $\begin{array}{c}0.400 \\
\{0.8491\}\end{array}$ \\
\hline
\end{tabular}

p-values in \{\} . AIC and SBC refer to Akaike and Schwarz information criteria, LL is the Log Likelihood, LBQ ${ }^{2}$ is the Ljung-Box test on squared standardized residuals 
Table 4. (cont.) Diagnostic tests of GARCH estimations

\begin{tabular}{|c|c|c|c|c|c|c|c|c|c|c|c|c|c|}
\hline Countries & $\mathrm{R}^{2} \mathrm{adj}$ & AIC & SBC & $\mathbf{L L}$ & $\mathrm{LBQ}^{2}(12)$ & $\begin{array}{l}\text { ARCH- } \\
\operatorname{LM(5)}\end{array}$ & Countries & $\mathrm{R}^{2}$ adj. & AIC & SBC & $\mathbf{L L}$ & $\mathrm{LBQ}^{2}(12)$ & $\begin{array}{l}\text { ARCH- } \\
\text { LM(5) }\end{array}$ \\
\hline $\begin{array}{c}\text { PERU } \\
\text { GJR-GARCH }\end{array}$ & -0.000233 & -6.367 & -6.327 & 7836.431 & $\begin{array}{l}12.074 \\
\{0.440\}\end{array}$ & $\begin{array}{c}1.095 \\
\{0.3605\}\end{array}$ & $\begin{array}{c}\text { THAILAND } \\
\text { GJR-GARCH }\end{array}$ & 0.005109 & -5.205 & -5.148 & 4207.102 & $\begin{array}{l}12.454 \\
\{0.410\}\end{array}$ & $\begin{array}{c}0.628 \\
\{0.6781\}\end{array}$ \\
\hline $\begin{array}{l}\text { PHILLIPINE } \\
\text { GJR-GARCH }\end{array}$ & 0.004395 & -5.790 & -5.752 & 7703.279 & $\begin{array}{l}21.180 \\
\{0.048\} \\
\end{array}$ & $\begin{array}{c}3.459 \\
\{0.0040\} \\
\end{array}$ & $\begin{array}{l}\text { TURKEY } \\
\text { GARCH }\end{array}$ & 0.005586 & -4.574 & -4.541 & 6081.203 & $\begin{array}{l}14.358 \\
\{0.278\}\end{array}$ & $\begin{array}{c}0.900 \\
\{0.4794\}\end{array}$ \\
\hline $\begin{array}{l}\text { PORTUGAL } \\
\text { GJR-GARCH }\end{array}$ & -0.005169 & -7.192 & -7.104 & 3386.642 & $\begin{array}{l}11.639 \\
\{0.475\}\end{array}$ & $\begin{array}{c}1.036 \\
\{0.3945\} \\
\end{array}$ & $\begin{array}{l}\text { UK-FTSE } 100 \\
\text { GJR-GARCH }\end{array}$ & 0.001180 & -6.583 & -6.564 & 20389.16 & $\begin{array}{l}25.488 \\
\{0.013\}\end{array}$ & $\begin{array}{c}3.255 \\
\{0.0061\} \\
\end{array}$ \\
\hline $\begin{array}{c}\text { RUSSIA } \\
\text { GJR-GARCH } \\
\end{array}$ & -0.000042 & -4.753 & -4.723 & 7783.382 & $\begin{array}{c}9.485 \\
\{0.661\} \\
\end{array}$ & $\begin{array}{c}0.352 \\
\{0.8808\} \\
\end{array}$ & $\begin{array}{c}\text { UK-FTSE } 250 \\
\text { EGARCH }\end{array}$ & 0.002036 & -6.793 & -6.749 & 7523.809 & $\begin{array}{c}8.577 \\
\{0.740\}\end{array}$ & $\begin{array}{c}0.448 \\
\{0.8149\} \\
\end{array}$ \\
\hline $\begin{array}{l}\text { SINGAPORE } \\
\text { GJR-GARCH }\end{array}$ & 0.005666 & -5.798 & -5.760 & 7714.159 & $\begin{array}{l}21.479 \\
\{0.044\}\end{array}$ & $\begin{array}{c}3.555 \\
\{0.0033\}\end{array}$ & $\begin{array}{c}\text { US DOW JONES } \\
\text { COMPOSITE } \\
\text { EGARCH }\end{array}$ & 0.001140 & -6.683 & -6.666 & 23424.66 & $\begin{array}{l}12.386 \\
\{0.415\}\end{array}$ & $\begin{array}{c}1.395 \\
\{0.2224\}\end{array}$ \\
\hline $\begin{array}{l}\text { SOUTH KOREA } \\
\text { GJR-GARCH }\end{array}$ & -0.000650 & -5.198 & -5.161 & 7203.808 & $\begin{array}{c}5.258 \\
\{0.949\} \\
\end{array}$ & $\begin{array}{c}0.386 \\
\{0.8584\}\end{array}$ & $\begin{array}{c}\text { US NASDAQ } 100 \\
\text { EGARCH }\end{array}$ & -0.000599 & -6.634 & -6.622 & 31522.97 & $\begin{array}{l}71.570 \\
\{0.000\}\end{array}$ & $\begin{array}{l}12.500 \\
\{0.000\}\end{array}$ \\
\hline $\begin{array}{c}\text { SPAIN } \\
\text { EGARCH }\end{array}$ & 0.000909 & -6.110 & -6.079 & 10239.49 & $\begin{array}{l}14.533 \\
\{0.268\}\end{array}$ & $\begin{array}{c}2.301 \\
\{0.0424\} \\
\end{array}$ & $\begin{array}{c}\text { US NEW YORK } \\
\text { COMPOSITE } \\
\text { EGARCH } \\
\end{array}$ & 0.000625 & -6.883 & -6.872 & 37052.48 & $\begin{array}{l}16.776 \\
\{0.158\} \\
\end{array}$ & $\begin{array}{c}2.369 \\
\{0.0217\} \\
\end{array}$ \\
\hline $\begin{array}{l}\text { SRI LANKA } \\
\text { EGARCH }\end{array}$ & 0.002140 & -6.644 & -6.604 & 8295.616 & $\begin{array}{l}21.645 \\
\{0.042\} \\
\end{array}$ & $\begin{array}{c}1.685 \\
\{0.1346\} \\
\end{array}$ & $\begin{array}{c}\text { US - S\&P } 500 \\
\text { EGARCH }\end{array}$ & 0.000722 & -6.936 & -6.927 & 51267.24 & $\begin{array}{l}26.926 \\
\{0.008\} \\
\end{array}$ & $\begin{array}{c}4.551 \\
\{0.0004\} \\
\end{array}$ \\
\hline $\begin{array}{c}\text { SWEDEN } \\
\text { GJR-GARCH }\end{array}$ & -0.002850 & -6.061 & -6.016 & 6095.232 & $\begin{array}{c}8.439 \\
\{0.750\} \\
\end{array}$ & $\begin{array}{c}0.612 \\
\{0.6902\}\end{array}$ & $\begin{array}{c}\text { YUGOSLAVIA } \\
\text { GJR-GARCH }\end{array}$ & 0.005578 & -6.173 & -6.069 & 2341.67 & $\begin{array}{c}8.176 \\
\{0.771\} \\
\end{array}$ & $\begin{array}{c}1.247 \\
\{0.2852\} \\
\end{array}$ \\
\hline $\begin{array}{c}\text { SWITZERLAND } \\
\text { EGARCH }\end{array}$ & 0.000447 & -6.456 & -6.432 & 14537.16 & $\begin{array}{c}1.142 \\
\{1.000\}\end{array}$ & $\begin{array}{c}0.043 \\
\{0.9989\} \\
\end{array}$ & $\begin{array}{l}\text { ZAMBIA } \\
\text { EGARCH }\end{array}$ & -0.003332 & -6.229 & -6.174 & 4902.757 & $\begin{array}{l}12.983 \\
\{0.370\} \\
\end{array}$ & $\begin{array}{c}0.270 \\
\{0.9293\} \\
\end{array}$ \\
\hline $\begin{array}{l}\text { TAIWAN } \\
\text { EGARCH } \\
\end{array}$ & 0.002320 & -5.618 & -5.581 & 7775.845 & $\begin{array}{l}19.689 \\
\{0.073\}\end{array}$ & $\begin{array}{c}2.973 \\
\{0.0111\} \\
\end{array}$ & & & & & & & \\
\hline
\end{tabular}

p-values in \{\} . AIC and SBC refer to Akaike and Schwarz information criteria, LL is the Log Likelihood, LBQ ${ }^{2}$ is the Ljung-Box test on squared standardized residuals 


\section{Appendix}

Programming routines for asymmetry tests in EVIEWS software

1. Sign Test

smpl@all

eq1.arch $(1,1)$ returns jan feb mar apr may jun jul aug sep oct nov dec eq1.makeresids resid01

GENR S_0 $=0$

SMPL@all IF resid01<0

GENR S_0 $=1$

smpl@all

eq1.1s resid01^2 c S_0

show eq1

2. Negative Bias Test

smpl@all

eq1.arch $(1,1)$ returns jan feb mar apr may jun jul aug sep oct nov dec eq1.makeresids resid01

GENR S_0 =0

SMPL@all IF resid01<0

GENR S_0 = 1

SMPL@äall

eq1.1s resid01^2 c S_0

GENR N_0 = S_0*resid01(-1)

eq1.ls resid01^2 c N_0

show eq1

3. Positive Bias Test

smpl@all

eq1.arch(1,1)returns jan feb mar apr may jun jul aug sep oct nov dec eq1.makeresids resid01

GENR S_0 $=0$

SMPL@all IF resid01<0

GENR S $0=1$

GENR N_0 $=$ S_0*resid01(-1)

GENR P_01 $=1-\bar{S} \_0$

GENR P_0 $=\mathrm{P}_{-} 01^{-} * \operatorname{resid} 01(-1)$

smpl@all

eq1.1s resid01^2 c P_0

show eq1 
4. Joint Test

smpl@all

eq1.arch $(1,1)$ returns jan feb mar apr may jun jul aug sep oct nov dec eq1.makeresids resid01

GENR S_0 $=0$

SMPL@all IF resid01<0

GENR S_0 $=1$

SMPL@all

eq1.1s resid01^2 c S_0

GENR N_0 $=$ S_0 $*$ resid01(-1)

GENR P $01=1-\bar{S} 0$

GENR P_0 $=$ P_0 ${ }^{-} * \operatorname{resid} 01(-1)$

smpl@all

eq1.1s resid01^2 c S_0 N_0 P_0

eq1. wald $\mathrm{c}(2)=0, \mathrm{c}(3)=0, \overline{\mathrm{c}}(4)=0$ 